\title{
Protagonists and spectators during photocatalytic solar water splitting with SrTaOxNy oxynitride
}

\section{Craig Lawley}

Paul Scherrer Institut https://orcid.org/0000-0003-4541-6600

\section{Zahra Pourmand Tehrani}

Paul Scherrer Institute

\section{Adam Clark}

Paul Scherrer Institute https://orcid.org/0000-0002-5478-9639

\section{Olga Safonova}

Paul Scherrer Institute https://orcid.org/0000-0002-6772-1414

\section{Max Doebeli}

ETH Zurich

\section{Vladimir Strocov}

Paul Scherrer Institute https://orcid.org/0000-0002-1147-8486

\section{Thomas Schmidt}

Electrochemistry Laboratory, Paul Scherrer Institute https://orcid.org/0000-0002-1636-367X

Thomas Lippert

Paul Scherrer Institute

\section{Maarten Nachtegaal}

Paul Scherrer Institute https://orcid.org/0000-0003-1895-9626

Daniele Pergolesi ( $\square$ daniele.pergolesi@psi.ch )

Paul Scherrer Institute https://orcid.org/0000-0002-6231-0237

\section{Article}

Keywords: Photoelectrochemical Cell, X-ray Absorption Spectroscopy, A-site Evolution, Surface Hydrophilicity, Operando Characterization

Posted Date: January 23rd, 2021

DOl: https://doi.org/10.21203/rs.3.rs-144979/v1

License: (c) (i) This work is licensed under a Creative Commons Attribution 4.0 International License. Read Full License 
Version of Record: A version of this preprint was published at Journal of Materials Chemistry A on September 21st, 2021. See the published version at https://doi.org/10.1039/D1TA05052D. 


\section{Title}

Protagonists and spectators during photocatalytic solar water splitting with $\mathrm{SrTaO}_{\mathrm{x}} \mathrm{N}_{\mathrm{y}}$ oxynitride.

\section{Authors}

Craig Lawley, ${ }^{\mathrm{a}, \mathrm{b}}$ Zahra Pourmand Tehrani, ${ }^{\mathrm{a}, \mathrm{b}}$ Adam H. Clark, ${ }^{\mathrm{a}}$ Olga V.

Safonova, ${ }^{a}$ Max Döbeli, ${ }^{c}$ Vladimir N. Strocov, ${ }^{a}$ Thomas J. Schmidt, ${ }^{\text {add }}$ Thomas Lippert, ${ }^{\text {a,bee }}$ Maarten Nachtegaal, ${ }^{\text {aa }}$ Daniele Pergolesi*a

\section{Author Affiliations}

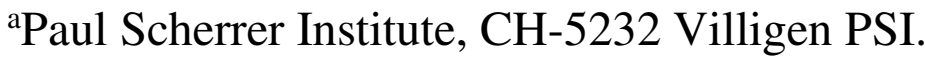

${ }^{b}$ ETH Zürich, Laboratory of Inorganic Chemistry, CH-8093 Zurich.

${ }^{c}$ ETH Zürich, Laboratory of Ion Beam Physics, CH-8093 Zurich.

${ }^{\mathrm{d}}$ ETH Zürich, Laboratory of Physical Chemistry, CH-8093 Zurich.

${ }^{\text {e}}$ International Institute for Carbon-Neutral Energy Research (I2CNER), Kyushu University, 744 Motooka 819-0395 Fukuoka, Japan

Correspondence to: Dr. D. Pergolesi (E-mail: daniele.pergolesi@psi.ch), Dr. M. Nachtegaal (E-mail: maarten.nachtegaal@psi.ch). 


\begin{abstract}
Oxynitrides have been shown to be promising visible light water splitting photocatalysts, but rapidly degrade under operating conditions. With a custom designed photoelectrochemical cell, we perform operando grazing incidence $\mathrm{X}$ ray absorption spectroscopy measurements on the oxynitride semiconductor SrTaOxNy during photocatalytic solar water splitting. We show that the nature of the A-site ( $\mathrm{Sr}$ ) and its evolution during operation, have large impacts on the overall stability and catalytic acitivity of the material, leading to an enriched BO2 $(\mathrm{Ta}(\mathrm{OH}) / \mathrm{TaO}(\mathrm{OH}))$ like surface. However, this usually beneficial effect with respect to increased surface hydrophilicity has complications for the efficiency of the photocatalytic process, as the $\mathrm{OH}$ and $\mathrm{O}(\mathrm{OH})$ intermediates formed are in competition between $\mathrm{O} 2$ generation and NOx species formation in the initial stages of operation. Operando characterisation of the evolution of the electronic structure of the photocatalyst proves to be an invaluable tool for the rational design and discovery of new and better performing materials.
\end{abstract}

\title{
Introduction
}

Independence from the reliance on fossil and nuclear fuels requires the sustainable production of green energy and chemicals. As an alternative, utilisation of solar energy is possible by several means: solar thermal applications, ${ }^{1}$ the direct conversion to electricity (photovoltaic), ${ }^{2}$ or storage in 
the form of chemical energy as solar fuels. ${ }^{3}$ One promising clean energy carrier and renewable fuel source is hydrogen harvested from photoelectrochemical (PEC) water splitting., , 5, 6

PEC water splitting requires the use of semiconductor (SC) photocatalysts that utilise solar light energy to generate electron/hole pairs. After reaching the surface, in contact with water, these charge carriers can be used to dissociate water molecules directly into molecular hydrogen and oxygen ${ }^{7}$. Perovskite oxynitrides are a promising class of SC materials for solar light driven water splitting. These materials have the general formula $\mathrm{ABO}_{3-\mathrm{x}} \mathrm{N}_{\mathrm{x}}$ (where $\mathrm{A}$ can be $\mathrm{La}, \mathrm{Sr}, \mathrm{Ba}, \mathrm{Ca}$, etc., and the $\mathrm{B}$ site: $\mathrm{Ti}, \mathrm{Ta}$, or $\mathrm{Nb}$ for example). The substitution of $\mathrm{N}$ into the $\mathrm{O}$ site of the precursor oxides affect the energy position of the band edges, reducing the band gap down to the visible light energy range. ${ }^{8}$ Moreover, in this class of compounds, the photogenerated electrons and holes, both possess enough energy to promote the hydrogen and oxygen evolution reaction, respectively.

The majority of studies to date focus on the characterisation of the photocatalytic properties of these materials in the form of powders, ${ }^{9,10,11}$ since powder development and optimisation is the primary way to device design and engineering. However, with powder samples, probing specific material properties is challenging. For example, the polycrystalline oxynitride powders 
do not provide well-defined surfaces to allow detailed studies of the solid-liquid interface, where the electrochemical reactions take place. The understanding of the catalytic process at the solid-liquid interface of oxynitride materials is therefore limited, as a result. A way to circumvent this limitation is the growth of thin films. By physical or chemical vapour deposition methods, it is possible to fabricate thin films with well-defined and atomically flat surfaces, therefore representing ideal model systems that allow the investigation of the surface and interface properties.

In this work, we present an operando study of the evolution of the photocatalyst semiconductor/water interface during photoelectrochemical solar water splitting using $\mathrm{SrTaO}_{\mathrm{x}} \mathrm{N}_{\mathrm{y}}(\mathrm{STON})$ thin films. Grazing incidence X-ray absorption spectroscopy (GIXAS) coupled with modulation excitation (ME)-XAS were used to increase the surface sensitivity, whilst probing epitaxially grown STON thin films with a custom designed operando reactor cell. This technique and experimental setup allow us to probe, during operation conditions, the changes of the local chemical and geometric environment surrounding the A- and Bcations of perovskite oxynitrides near and at the solid-liquid interface. Due to the attenuation of X-rays by the aqueous electrolyte, it is not feasible to extend this study to the $\mathrm{O}$ and $\mathrm{N}$ anions, since the $\mathrm{O} 1 \mathrm{~s}$ and $\mathrm{N} 1$ s edges are too low in energy (543.1 eV and $409.9 \mathrm{eV}$, respectively). Therefore, complimentary ex-situ X-ray photoelectron spectroscopy (XPS) has been included to observe all four 
elements $(\mathrm{Sr}, \mathrm{Ta}, \mathrm{O}$, and $\mathrm{N})$ in their initial and final states. With this experimental strategy, we are able to show that it is possible to achieve a better understanding of the physicochemical processes occurring at the oxynitrideliquid interface, leading to a degradation of the photocatalytic activity. Our findings can be of great importance for the rational design of stable oxynitride SC's for solar water splitting.

\section{Results}

\section{Photoelectrochemical Water Splitting}

The splitting of water into molecular $\mathrm{H}_{2}$ and $\mathrm{O}_{2}$ is an energetically unfavourable reaction. It requires a standard Gibbs free energy change of $+237.2 \mathrm{~kJ} \mathrm{~mol}^{-1}$, which also corresponds to a potential of $1.23 \mathrm{eV}$ per electron. Moreover, for both half reactions of the solar water splitting process to proceed, that is the hydrogen evolution reaction (HER) and oxygen evolution reaction (OER), first the kinetic overpotentials must be overcome. Therefore, a suitable SC material for visible light PEC water splitting should have (a) a band gap $\left(E_{g}=E_{C B}-E_{V B}\right)$ in the range of $1.6-2.4 \mathrm{eV}$ to sufficiently utilise the solar spectrum and drive the reactions, and (b) energy position of the band edges appropriately aligned with respect to the HER and OER redox potentials. 
As mentioned above, a number of oxynitride perovskite materials possess these

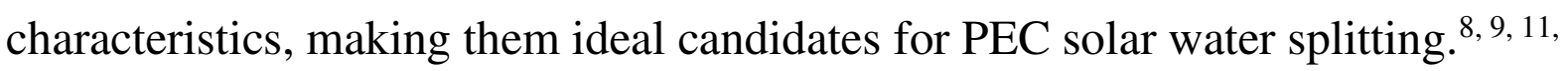
${ }^{12,13}$ Thin films of these materials provide excellent model systems to probe the physical and chemical evolution of the surface of the $\mathrm{SC}$, in contact with water. Fig. 1 shows the energy diagram schematic for PEC water splitting using a STON thin film as a light harvesting SC photocatalyst and TiN as the current collector. In Fig. 1a, we see that upon light irradiation with photons with an energy $(\mathrm{hv})>1.23 \mathrm{eV}$, the photon is absorbed promoting a photoexcited electron ( $\left.\mathrm{e}^{-}\right)$from the valence band (VB) to the conduction band (CB). The photoelectrons travel via a TiN current collector layer and external circuit where they are involved in the HER using a platinum counter electrode (cathode). The generation of a photoelectron leaves behind a photogenerated electron hole $\left(\mathrm{h}^{+}\right)$ which migrates towards the surface of the SC oxynitride where it is then involved in the OER.

Fig. $1 \mathrm{~b}$ shows the three-electrode configuration used in this work, where the STON SC photocatalyst is used as a photoanode to study the OER, a platinum counter electrode used for the HER half reaction, and a $\mathrm{Ag} / \mathrm{AgCl}$ reference electrode is used to control the applied potential on the working electrode. The electronic current between the working and counter electrodes is called the photocurrent and the photocurrent is proportional to the amount of molecular $\mathrm{H}_{2}$ and $\mathrm{O}_{2}$ produced. The photocurrent is defined as the current response under 

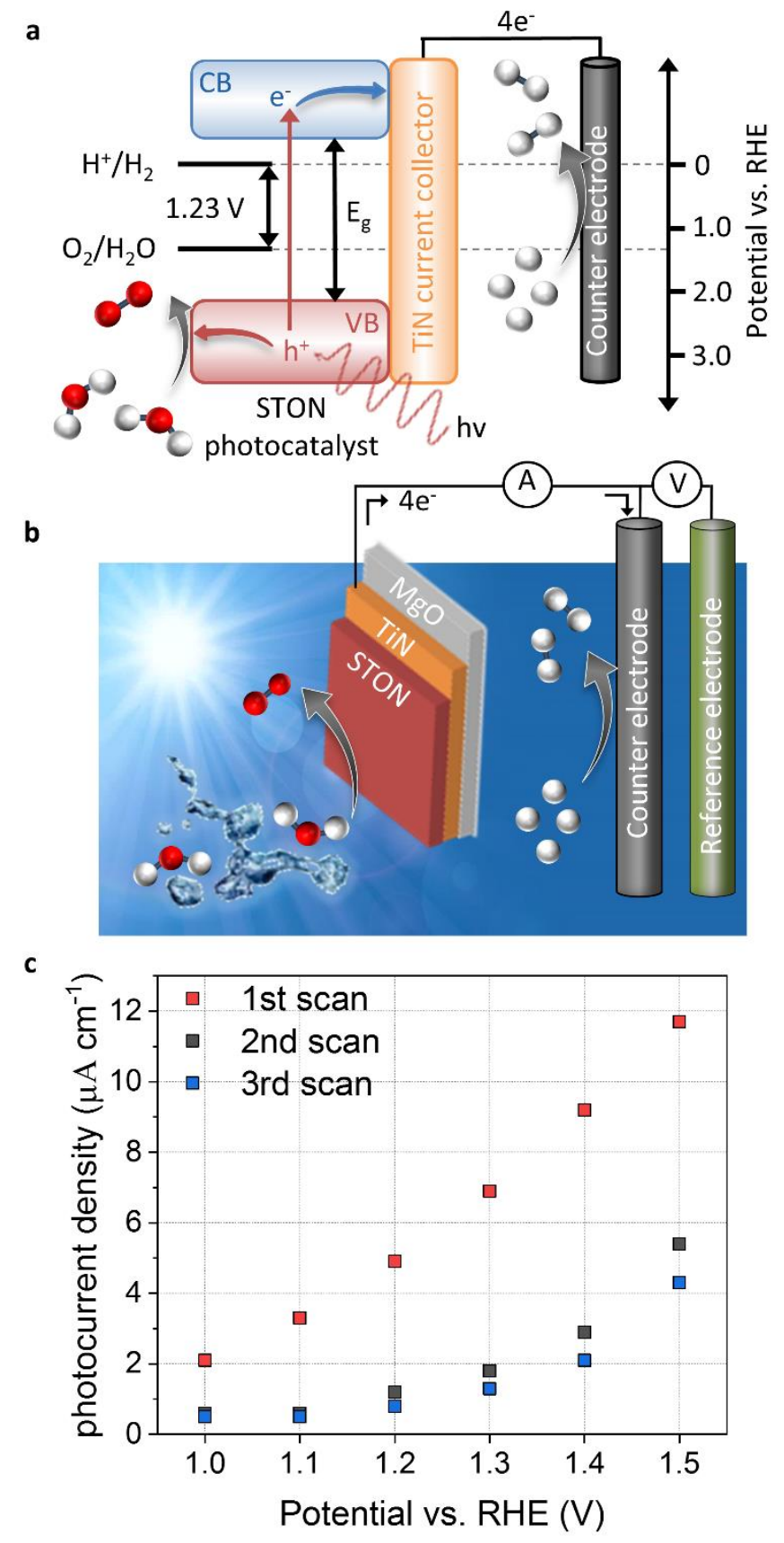

Figure 1. Photoelectrochemical characterisation (a) Energy diagram for photocatalytic water splitting, (b) experimental schematic of the photoelectrochemical three electrode cell. The working, counter and reference electrodes are the oxynitride thin film, platinum wire and $\mathrm{Ag} / \mathrm{AgCl}$ respectively, 
(c) photocurrent densities for STON for the first three potentiodynamic measurements.

light conditions minus the current response under dark conditions. More indepth experimental details are included in the corresponding methods section. Fig. 1c shows the PEC performance of the STON oxynitride thin films, the initial photocurrent reaches a photocurrent density of ca. $12 \mu \mathrm{A} \mathrm{cm}^{-1}$ at $1.5 \mathrm{~V}$ vs. reversible hydrogen electrode (RHE). This value is in line with previous reports on oxynitride thin films using the bare SC material without surface decoration with co- catalyst, which dramatically facilitate hole extraction. On the one hand, the $\mathrm{N}$ content of textured thin films is typically lower than the stoichiometric value that can be obtained with powder samples. ${ }^{14}$ This reduces the thin films photo-response to visible light illumination. On the other, the thin films possess atomically flat surfaces compared to the corresponding oxynitride powders. Therefore, the surface area is ca. 20 times smaller than it would be in their powder forms. ${ }^{15}$ However, after successive measurements (potentiodynamic sweeps) the material shows significant degradation in its initial performance.

The stabilised photocurrent density shows values ca. $40 \%$ of its initial value. The sudden and large degradation in performance is a huge hindrance for the application of a material that initially seems quite promising. Some studies have looked at ways of improving the performance and long-term stability of 
STON $^{10,16,17}$ by doping constituent elements and/or decorating the surface with co-catalyst nanoparticles. However, probing the physicochemical evolution of the surface under operation conditions will provide insight into what physical and chemical processes occur at the surface during the OER and therefore, allow a rational design of stable STON.

\section{Oxynitride Thin Films}

The STON thin films were fabricated using a modified pulsed laser deposition technique described in previous works, ${ }^{18}$ followed by post annealing in $\mathrm{NH}_{3}$ for 1 hour. The epitaxial oxynitride STON films were deposited on titanium nitride (TiN) coated magnesium oxide single crystal substrates (001) oriented. Rutherford back scattering (RBS) and elastic recoil detection analysis (ERDA) determined the chemical compositions of the STON thin films as $\mathrm{Sr}_{0.94} \mathrm{Ta}_{1.06} \mathrm{O}_{2.80} \mathrm{~N}_{0.31}$. The experimental uncertainties for $\mathrm{Sr}$ and $\mathrm{Ta}$ are $\pm 2 \%$ (RBS) and $\pm 7 \%$ for $\mathrm{O}$ and $\mathrm{N}$ (ERDA). The full results have been included in supplementary Fig. 1 and supplementary Table 1. The O:N ratio of ca. 10 is in line with previous examples of highly ordered oxynitride thin films, since there is a trade-off between overall nitrogen content and crystalline quality, ${ }^{19}$ where films with larger $\mathrm{N}$ contents tend to be more disordered. 
From Fig. 2 it can be seen that the TiN buffer layer grows (001) epitaxially oriented on the $\mathrm{MgO}$ substrate. The (002) reflex of $\mathrm{TiN}$ is visible as a shoulder on the left hand side of the peak of the substrate (see supplementary Fig. 2). Fig. 2 also shows that STON grows epitaxially on the TiN buffer layer ${ }^{18}$ with the (hkl) reflexes (002) and (004) appearing at $2 \theta$ values of ca. $21.8^{\circ}$ and $44.2^{\circ}$, respectively. The angular position of the (001) reflexes of the perovskite $\mathrm{SrTaO}_{2} \mathrm{~N}$ have been marked in blue as reference from the Inorganic Crystal Structure Database (ICSD).

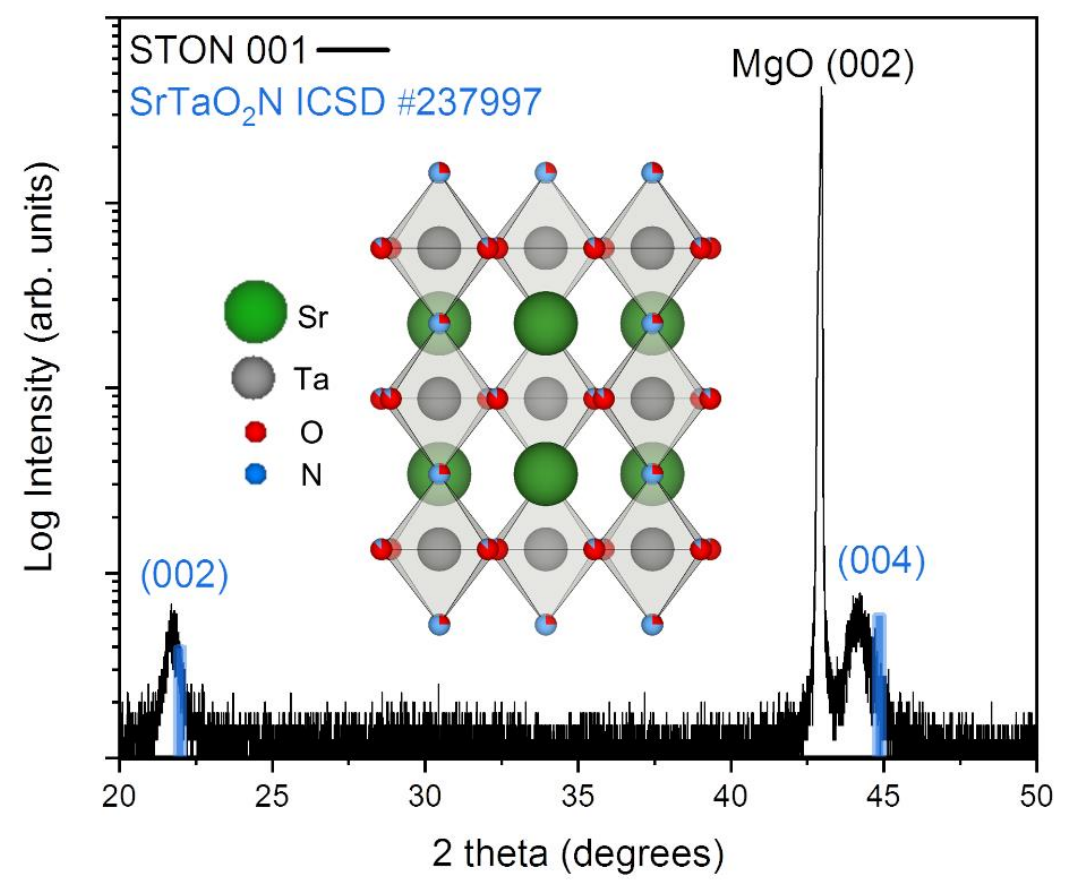

Figure 2. XRD pattern of the epitaxially grown STON film. The $\theta / 2 \theta$ scan is shown in black, with the angular position of the (001) reflexes of $\mathrm{SrTaO}_{2} \mathrm{~N}$ shown in blue for reference. The inset shows the crystal structure of $\mathrm{SrTaO}_{2} \mathrm{~N}$. 
Both the 002 and 004 reflexes of the STON thin film are slightly shifted to lower $2 \theta$ values compared to the $\mathrm{SrTaO}_{2} \mathrm{~N}$ reflexes. This is due to the combined effect of a difference in $\mathrm{N}$ content to the stoichiometric reference and the crystalline constrain (lattice mismatch between the substrate and film) induced by the TiN-coated $\mathrm{MgO}$ substrate. The perovskite structure of STON remains stable, within a large range of $\mathrm{N}$ content. However, the cell parameters can be significantly affected since the $\mathrm{N}$ content affects the Ta-O-Ta dihedral bond angle and ultimately, the overall distortion of the cell. To characterise how the OER modifies the physicochemical properties of the surface of our STON films, we first used XPS to compare changes of the oxidation state of all four elements before and after PEC testing.

\section{X-ray Photoelectron Spectroscopy}

The XPS spectra for all four elements are shown in Fig 3. For all Sr spectra collected after PEC, the peaks shift to lower energy by ca. $0.10-0.15 \mathrm{eV}$, as can be seen in Fig. 3a and 3b, suggesting an increase in electron density surrounding $\mathrm{Sr}$ (formally $2+$ in STON). This shift in binding energy is likely associated with the coupling of oxygen adsorbates on the Sr sites, which then desorb 
contributing to $\mathrm{O}_{2}$ generation, leaving the once occupied site vacant on $\mathrm{Sr}$.

Where deprotonation was calculated as the overpotential determining step
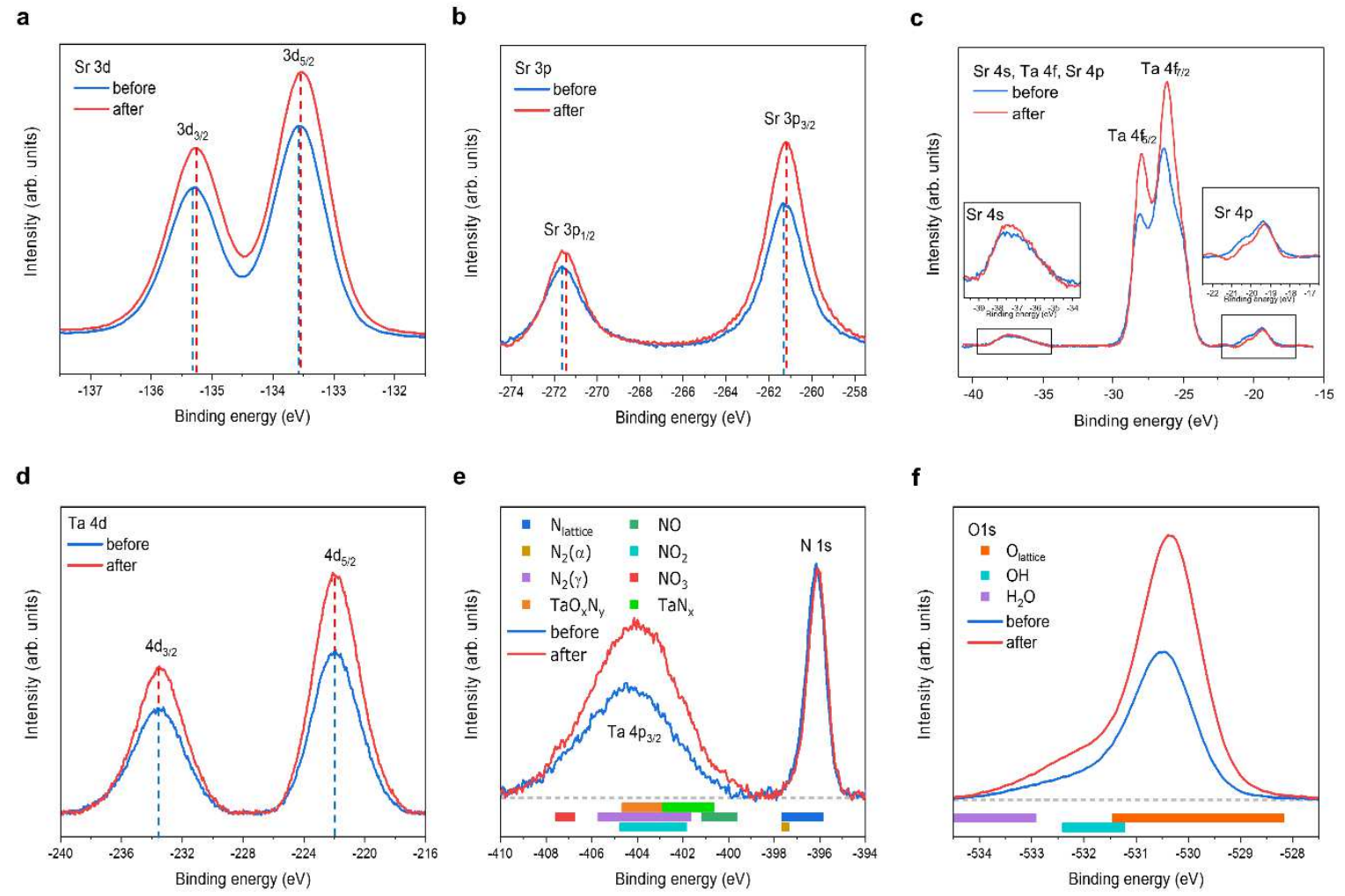

Figure 3. STON thin film XPS spectra before and after PEC. (a) $\mathrm{Sr} 3 \mathrm{~d}$

spectra, (b) Sr 3p spectra, (c) Sr 4s, Sr 4p and Ta 4f spectra, (d) Ta 4d spectra, (e) Ta $4 p$ and $\mathrm{N}$ 1s spectra, (f) O 1s spectra. Before and after PEC shown in blue and red, respectively. Reported binding energies for the associated chemisorbed $\mathrm{N}$ and $\mathrm{O}$ species are included in parts (e-f) to clarify the convolution of the $\mathrm{N} 1 \mathrm{~s}$ and Ta 4p3/2 and the shoulder of the $\mathrm{O}$ 1s spectra, respectively. References are included in the supplementary. 
(ODS) for Sr. ${ }^{20}$ Under ambient conditions/aqueous environment, $\mathrm{Sr}$ should be covered in $\mathrm{OH}$ adsorbates. After deprotonation (slow step) the OER proceeds, leaving vacant oxygen cavity on $\mathrm{Sr}$, assuming the traditional 4-step mechanism for the OER. ${ }^{21}$ However, SrO is partially soluble in alkaline electrolyte (pH13) to form ionic $\mathrm{Sr}^{2+}$ and $2 \mathrm{OH}^{-}$. $\mathrm{OH}$ adsorbates would increase the coordination on the lattice $\mathrm{Sr}$ site This, in conjunction with the reductive shift in binding energy, would decrease the lattice energy (ionic bond strength estimation). Therefore, dissolution and leaching of $\mathrm{Sr}^{2+}$ into the electrolyte would be expected. Especially, in an alkaline medium such as $\mathrm{NaOH} / \mathrm{H}_{2} \mathrm{O}$ with a $\mathrm{pH}$ of 13 . Examples of lattice energies for $\mathrm{Sr}^{2+}$ in $\mathrm{Sr}(\mathrm{OH})_{2}, \mathrm{Sr}\left(\mathrm{NO}_{3}\right)_{2}$ and $\mathrm{SrO}$ are 2330, 2176 and $3217 \mathrm{~kJ} \mathrm{~mol}^{-1}$, respectively (values taken from $\mathrm{Ref}^{22}$ ). Although these are reference values, we can see how the changes to the Sr environment can affect ionic cohesive forces and hence, the stability.

After PEC, we see an overall increase in peak area and intensity for the Sr XPS spectra. It has previously been shown, that an increase in XPS peak intensity can result from $\mathrm{Sr}$ segregation/accumulation ${ }^{23,24}$ as well as, increased doping concentrations. ${ }^{25}$ Peak intensity/area increases are also observed due to $\mathrm{Sr}$ particle/Sr surface species formation. ${ }^{23,24}$ In this work however, the increases in peak area and intensity are likely due to the increase in surface hydrophilicity (Fig. 3f). The Sr XPS main peaks include an average of $\mathrm{SrO}, \mathrm{SrOH}$ and $\mathrm{Sr}$ sites 
with vacant oxygen cavities all contributing to the total peak areas. The vacant cavity site would de-shield Sr so that it appears enriched, and the changing ratios of the average states would account for the shift in position and increases in intensities.

Fig. 3c includes the $\mathrm{Sr} 4 \mathrm{~s}$, Ta 4f, and $\mathrm{Sr} 4 \mathrm{p}$ edges with decreasing binding energy. Ta $4 \mathrm{f}$ spectra exhibit two peaks due to the Ta $4 f_{5 / 2}$ and $4 f_{7 / 2}$ contributions. The peak fitting analysis suggests that there are two Ta binding environments corresponding to $\mathrm{TaO}_{\mathrm{x}}$ and $\mathrm{TaO}_{\mathrm{x}} \mathrm{N}_{\mathrm{y}}$ like environments (supplementary Fig. 3a-c). After PEC, the peak contributions of $\mathrm{TaO}_{\mathrm{x}} \mathrm{N}_{\mathrm{y}}$ decrease and those of $\mathrm{TaO}_{\mathrm{x}}$ increase, suggesting a slight loss of nitrogen and an increase in the overall oxygen content at the surface. The energy positions of the peaks suggest a slight reductive shift by ca. $+0.15 \mathrm{eV}$. However, the Ta $4 \mathrm{~d}$ spectra in Fig. 3d show an increase in the FWHM and peak intensities, but no shift in energy position or peak separation. The shift in the Ta 4f spectra may then also be related to the changing $\mathrm{O} / \mathrm{N}$, resulting in disorder and differences in the charge transfer between the Ta $4 \mathrm{f}$ final states and the $\mathrm{O} 2 \mathrm{p} / \mathrm{N} 2 \mathrm{p}$ orbitals. This then shifts the mid gap $\mathrm{f}$ band higher towards the $\mathrm{CB}$ or above,${ }^{26}$ leaving the $4 \mathrm{~d}$ core states unchanged. The increase in intensity observed could be a result of apparent $\mathrm{Ta}$ enrichment due to the $\mathrm{O} / \mathrm{N}$ vacancy generation exposing the subsurface B sites. ${ }^{27}$ It could also be a result of Sr leaching, also exposing the subsurface Ta on average, Since Sr is more soluble than Ta and, it has been 
shown that Ta is passive in alkaline electrolytes with concentrations $2.5 \mathrm{x}$ stronger than used in this work. ${ }^{28}$

The Ta $4 p_{3 / 2}$ is observed as a broad peak at ca. $404-405 \mathrm{eV}$ (Fig. 3e). However, the Ta $4 p_{3 / 2}$ spectrum overlaps with the $\mathrm{N} 1 \mathrm{~s}$ signal in the energy region at ca. 402-408 eV which contains the $\mathrm{N}$ 1s information regarding chemisorbed NO, $\mathrm{NO}_{2}, \mathrm{NO}_{3}$ and $\mathrm{N}_{2}$, making the peak analysis difficult. The $\mathrm{N} 1$ s Ta-N lattice signal can be seen as a sharp peak at ca. $396.1 \mathrm{eV}$. After PEC, the main N 1s peak shows a slight reductive shift by ca. $0.1-0.15 \mathrm{eV}$. This reductive shift is likely related to the slight loss of $\mathrm{N}$ from the lattice and/or the formation of $\mathrm{NO}_{\mathrm{x}}$ species, with a slight change in electron density as a result. The broad peak in the region, which contains the convoluted $\mathrm{N} 1 \mathrm{~s}$ and $\mathrm{Ta} 4 \mathrm{p}_{3 / 2}$ signals increases significantly after PEC suggesting an increase in the number of chemisorbed $\mathrm{N}$ states and Ta enrichment after PEC. However, the peak contributions cannot be resolved with confidence due to the superimposition of the Ta $4 \mathrm{p}$ and $\mathrm{N} 1 \mathrm{~s}$ signals. At ca. $407.5 \mathrm{eV}$ there is a small but obvious increase in intensity belonging to a different $\mathrm{Ta} / \mathrm{N}$ binding state, which is likely a contribution due to chemisorbed $\mathrm{NO}_{3}$ according to literature reports (supplementary table 5). It is therefore assumed that $\mathrm{NO}$ and $\mathrm{NO}_{2}$ would contribute to the overall increased intensity/peak area observed, where the $\mathrm{O}(\mathrm{OH})$ OER intermediate formed during the reaction likely oxidised the $\mathrm{NO}_{\mathrm{x}}$ species in competition with $\mathrm{O}_{2}$ 
evolution. This would reduce the overall efficiency of the material, and chemisorbed $\mathrm{NO}_{\mathrm{x}}$ species would occupy possible active sites. This, in conjunction with Sr segregation, could explain the large degradation in initial photocurrent observed.

With respect to oxygen, after PEC the O 1s spectra (Fig. 3f) exhibits a large increase in the intensity of the main peak and shoulder feature(s), accompanied by an increase in the FWHM and a reductive shift (ca. $0.15-0.2 \mathrm{eV}$ ) of the main peak situated at ca. $530.4 \mathrm{eV}$. The shoulder feature is observable on the highenergy side of the asymmetric $\mathrm{O}$ 1s lattice peak at ca. $531.5-534 \mathrm{eV}$. A comparison of the peak position to references (Fig. $3 \mathrm{f}$ bottom) suggests a minimum of two binding environments, likely associated with chemisorbed $\mathrm{OH}$ and/or $\mathrm{H}_{2} \mathrm{O}$ species (Fig. 3f). Peak fitting was not performed, due to the uncertainty and superimposition of the $\mathrm{O}$ and $\mathrm{NO}_{\mathrm{x}}$ species. In addition, after PEC the integrated area under the shoulder increases. This is due to the increased hydrophilicity of the surface. This, together with the suggested changes in N/O, agrees with a larger overall $\mathrm{O}$ surface content after PEC. The increased $\mathrm{O}$ content suggests that any vacancies generated at the surface/surface layers are self-healed under OER conditions. ${ }^{29}$

Clearer evidence of $\mathrm{NO}_{x}$ formation for the oxynitride $\mathrm{LaTiO}_{x} \mathrm{~N}_{\mathrm{y}}$ can be seen by XPS in a previous work ${ }^{19}$, where Ta does not convolute the $\mathrm{N}$ 1s signal. We also 
observe evidence for the depletion of $\mathrm{N}$ states from the lattice structure using angle-resolved photoemission spectroscopy (ARPES) ${ }^{30}$ A more detailed discussion on $\mathrm{N}_{2} / \mathrm{NO}_{\mathrm{x}}$ formation is included in the discussion section.

Overall, the XPS analyses of the initial and final state of the STON photocatalyst suggest that STON suffers from a surface degradation and reconstruction, which lead to a dramatic decrease in photocurrent (Fig. 1c). This process involves (a) loss of $\mathrm{N}$ from the structure (including interstitial $\mathrm{N}$ ), where $\mathrm{N}$ remains partially chemisorbed as $\mathrm{N}_{2} / \mathrm{NO}_{x}$ species, (b) an increase in electron density on $\mathrm{Sr}$ (c) Ta enrichment/exposure with an increase in disorder of its local environment due to changes in hybridisation with the $\mathrm{N}$ and $\mathrm{O} 2 \mathrm{p}$ states. Next, we explore the effect of several external stimuli (applied potential and light) on the surface of the oxynitride SC under oxygen evolution reaction conditions by operando XAS in liquid phase.

\section{Operando Reactor Cell}

The operando XAS measurements were performed in a custom-built reactor cell designed and fabricated at the Paul Scherrer Institute for the specific purpose of operando GIXAS in aqueous electrolytes for thin films. The cell design is schematically depicted in Fig. 4. The cell is made of polyether ether ketone 
(PEEK), a thermoplastic with high mechanical and chemical stability, allowing the use of most common acidic/alkali based aqueous electrolytes. The X-ray beam illuminates the surface of the sample at grazing angle and the fluorescent signal is detected as a cone along the surface normal, as shown in Fig. 4a. The surface sensitivity that can be achieved in grazing angle geometries considering the incident energy of the X-rays is ca. $3 \mathrm{~nm}^{31}$

a

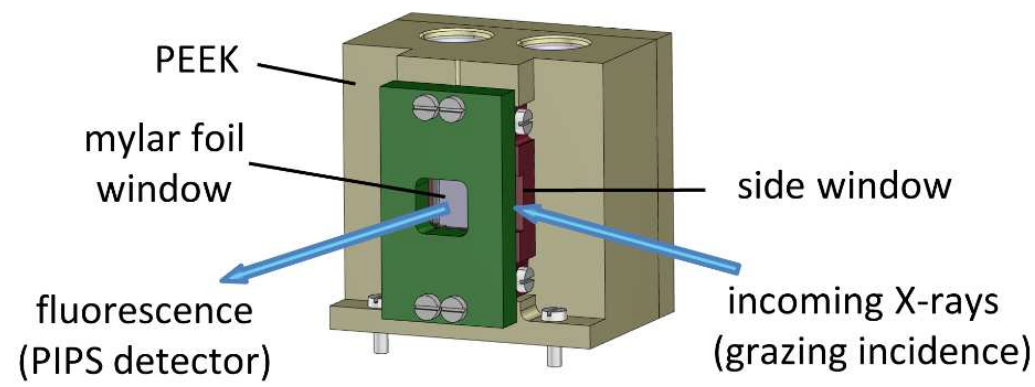

b Electrode holders

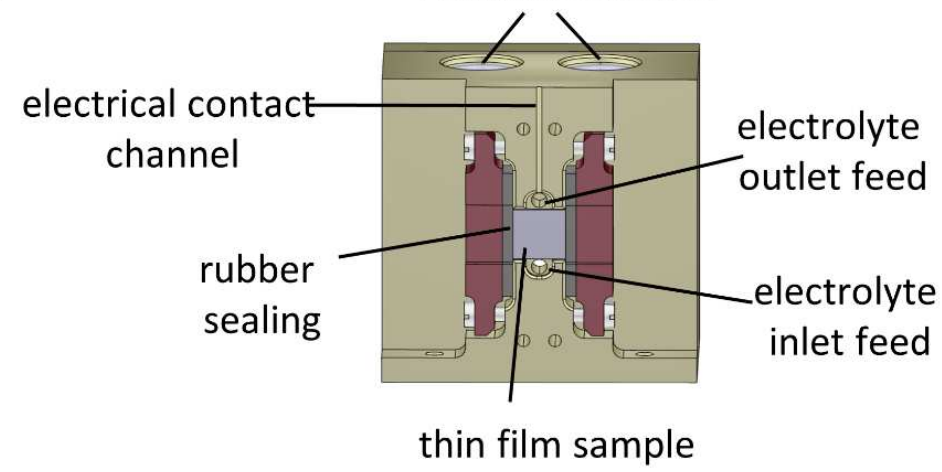

Figure 4. Operando reactor cell for surface sensitive GIXAS measurements.

(a) trimetric view of the cell and GIXAS geometry used during measurements, where the grazing incident X-rays enter through a Mylar foil covered side window, (b) cross sectional internal view. Where, PIPS denotes a passivated implanted planar silicon detector. 
The cell is designed to hold thin films grown onto standard substrate sizes. Typically, $10 \times 5 \mathrm{~mm}$ or $10 \times 10 \mathrm{~mm}$ with a substrate thickness of $0.5-1.0 \mathrm{~mm}$. The window material used is an optically clear Mylar foil $(\mathrm{t}=0.19 \mathrm{~mm})$, which allows the transmission of visible light onto the sample whilst also allowing > $90 \%$ X-ray transmission at the energy ranges used in this work (supplementary Fig. 4). The use of Mylar foil as a window provides flexibility in enabling visible light to shine onto the sample whilst detecting the fluorescence signal, as well as allowing grazing incidence angles of $<1$ degree onto the sample surface in an aqueous environment. Synthetic rubber pieces clamped into position seal the Mylar foil window to prevent leaks (Fig. 4b). On the backside (supplementary Fig. 5) are two threaded openings, allowing the use of an external electrolyte reservoir as well as connecting a peristaltic pump or a syringe pump system to circulate the electrolyte as required. Alternatively, the cell can use an internal electrolyte reservoir situated behind the sample. The cells' internal reservoir can house both the counter and reference electrodes.

\section{Tantalum $L_{3}$ Edge XANES}

Fig. 5a shows the XANES spectra recorded ex-situ at the Ta $\mathrm{L}_{3}$ edge $\left(\mathrm{E}_{0}=9881\right.$ $\mathrm{eV}$ ) for $\mathrm{BaTaO}_{2} \mathrm{~N}(\mathrm{BTON}), \mathrm{STON}$, and $\mathrm{KTaO}_{3}$ powder samples. These 
measurements are used as reference to understand the corresponding GIXAS measurements performed during OER using the STON thin films, as shown in Fig. 5b. We use $\mathrm{KTaO}_{3}$ as a reference for this work since the parent oxide of STON, which would be $\mathrm{Sr}_{2} \mathrm{Ta}_{2} \mathrm{O}_{7}$, possesses a different crystal structure,
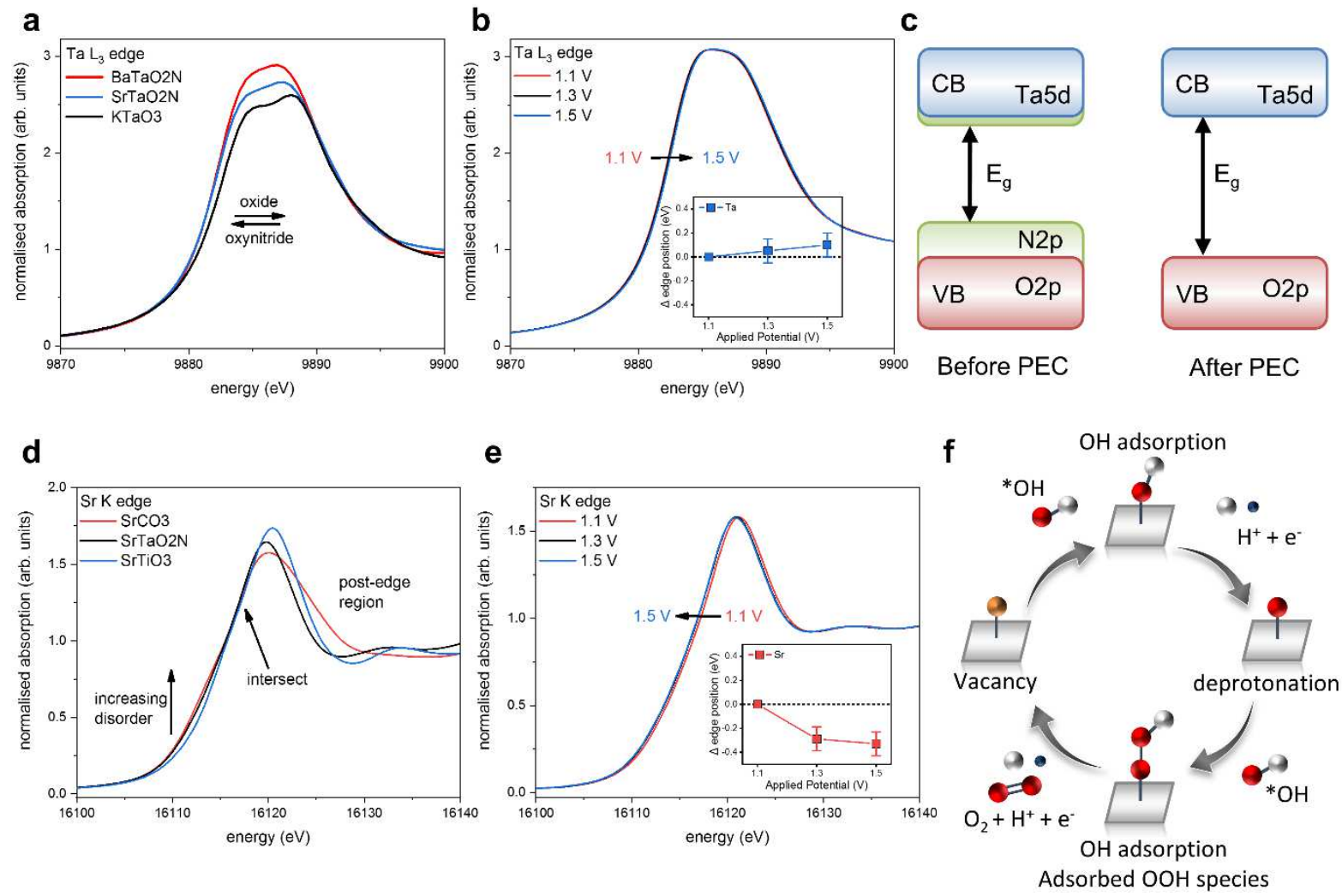

Figure 5. Operando GIXAS at the STON-liquid interface (a) Reference spectra for $\mathrm{Ta}^{5+}$ oxide and oxynitride powders, (b) operando measurements for STON thin film during the chronoamperometry (1 hour) measurements, with stepped applied potential. The error bars included in the magnified insert correspond to $\pm 0.1 \mathrm{eV}$, (c) graphic representation for the suggested changes occurring to the band structure of the surface layers of the STON photocatalyst. $\mathrm{VB}, \mathrm{CB}$ and $\mathrm{E}_{\mathrm{g}}$ denote the valence band, conduction band and band gap, 
respectively, (d) reference spectra for $\mathrm{Sr}$ containing powders, (e) operando measurements for STON thin film during the chronoamperometry (1 hour) measurements, with stepped applied potential. The error bars included in the magnified insert correspond to $\pm 0.1 \mathrm{eV}$, (f) suggested changes with respect to Sr after PEC.

Therefore, the electronic structure would differ significantly. $\mathrm{KTaO}_{3}$ has a cubic crystal structure like $\mathrm{SrTaO}_{2} \mathrm{~N}$ and $\mathrm{BaTaO}_{2} \mathrm{~N}$. Moreover, in all three cases Ta exists in the 5+ oxidation state, therefore allowing a sensible comparison of their electronic structures (Fig. 5a).

We can see that, compared to $\mathrm{KTaO}_{3}$, for the oxynitride powders there is a shift of the edge position $\left(\mathrm{E}_{0}\right)$ to lower energies by ca. $0.5 \mathrm{eV} \pm 0.1$. This shift of the absorption edge is not due to the reduction of Ta but rather due to the downward shifting of the $\mathrm{CB}$ minimum of STON and BTON compared to $\mathrm{KTaO}_{3}$. The $\mathrm{CB}$ is comprised of Ta $5 \mathrm{~d}$ orbitals for all three materials. ${ }^{20}$ The substitution of nitrogen into the oxygen sites affects the Ta-O/N-Ta dihedral bond angle and the overall electronegativity of the anions. Both these effects can explain the downward shift in energy of the $\mathrm{CBM}^{32,33}$ schematically depicted in Fig. 5c. The increase in intensities for the XANES spectra most likely result from the nitrogen substitution. In the cubic perovskite oxide $\mathrm{KTaO}_{3}$, the Ta-O-Ta angle is $180 \mathrm{deg}$. In the orthorhombic oxynitride perovskites, the dihedral bond angle is 
distorted and its value depends on the total $\mathrm{N}$ content and the size of the $\mathrm{A}$ cation. Therefore, with the lowering of symmetry and increased p-d orbital mixing, the $2 p-5 d$ transition will be more favourable and reflected in the relative intensities of the peaks.

Fig. 5b shows the operando XANES measurements performed on STON thin films during the chronoamperometry measurements (measuring the photocurrent at a fixed applied potential over time). We can see that for all applied potentials, there is no significant change in the spectral shape or in the edge position, indicating no change in formal oxidation state. When inspecting the magnified inset, there are small shifts in the edge positions to higher energies when moving from 1.1 to 1.3 and 1.3 to $1.5 \mathrm{~V}$ vs. RHE, corresponding to ca. 0.05 and $0.1 \mathrm{eV}$ respectively. It has previously been shown that for various compounds which all contain formally $\mathrm{Ta}^{5+}$ species, the edge position can vary by ca. $1.4 \mathrm{eV}^{34}$ due to the variation of the electronic structure and the relative position of the Ta $5 \mathrm{~d}$ states. Therefore, the small shifts seen in edge position for Ta are unlikely to be oxidation of Ta at the surface. Even though oxidation states are often valid interpretations due to the correlation between formal oxidation state and edge position, ${ }^{35,36,37}$ the changes shown here are first within error and second any small change is likely due to the slight loss of lattice nitrogen and/or the possible formation of $\mathrm{NO}_{\mathrm{x}}$ species at the surface of 
the STON thin film as suggested by the XPS analysis in this work. As previously discussed, upon partial $\mathrm{N}$ loss in the surface layer, it will also result in a local loss of hybridisation between the Ta $5 \mathrm{~d}$ and the $\mathrm{N} 2 \mathrm{p}$ states at the VB maximum (Fig. 5c). As a result, a more 'oxide-like' electronic structure forms at the surface as the potential is increased.

Iis reasonable to assume that Ta will tend to fulfil its full coordination sphere upon loss of $\mathrm{N}$ by replacing with $\mathrm{O}$ under OER conditions. ${ }^{29}$ This would also explain why there is no significant change in the spectral shape for Ta. There is instead a slight shift to higher energy of the absorption edge, which is commonly assumed to be resulting from oxidation. ${ }^{35,38}$ However, the replacement of $\mathrm{N}^{3-}$ with $\mathrm{O}^{2-}$ should see a reductive shift to lower energy for Ta. This again suggests that the charge around Ta is stabilised, like for Ti in $\mathrm{LaTiO}_{\mathrm{x}} \mathrm{N}_{\mathrm{y}}{ }^{31}$, but due to slight changing $\mathrm{O} / \mathrm{N}$ concentrations at the surface, the Ta octahedra undergo a slight disorder. Since the CBM is comprised of delocalised Ta $5 \mathrm{~d}$ states in $\mathrm{STON}^{39}$, the resulting changes in the $\mathrm{O} / \mathrm{N}-\mathrm{Ta}-\mathrm{O} / \mathrm{N}$ dihedral bond angle and degree of hybridisation, affect the conduction band minimum position. ${ }^{33}$

\section{Strontium K edge XANES}


Fig. 5d shows the XANES spectra measured at the $\mathrm{Sr} \mathrm{K}$ edge $\left(\mathrm{E}_{0}=16105 \mathrm{eV}\right)$ for $\mathrm{Sr}$ containing oxide $\left(\mathrm{SrTiO}_{3}\right)$, carbonate $\left(\mathrm{SrCO}_{3}\right)$ and oxynitride (STON) powders. Small changes in the post edge region and in the intensities of the main peak (16120-16140 eV) can be observed and are due to the differences in coordination environment surrounding the Sr cations. ${ }^{40}$ There are also changes of intensity of a broad shoulder in the pre-edge region at ca. $16108-16115 \mathrm{eV}$. Pre edge features relate to changes in the local geometry and degree of disorder surrounding the absorbing element of interest. Therefore, as one moves away from a perfect octahedral environment the pre-edge intensities increase ${ }^{41}$ and this is observed from the perfect octahedral environment surrounding $\mathrm{Sr}$ in $\mathrm{SrTiO}_{3}$, to the increasingly disordered octahedra in $\mathrm{SrTaO}_{2} \mathrm{~N}$ and $\mathrm{SrCO}_{3}$, respectively.

Fig. 5e shows the operando XANES measurements performed on the STON films during the chronoamperometry measurements as described previously. Unlike for the Ta B site, here we observe a more significant shift in the edge position due to an increase of electron density on $\mathrm{Sr}^{2+}$, with increasing the applied potential from 1.1 to $1.3 \mathrm{~V}$ vs. RHE. Increasing the potential further from $1.3 \mathrm{~V}$ to $1.5 \mathrm{~V}$ does not show any further significant modification of either the local coordination or electronic structures around the Sr cations. The observed shift in edge position with potential is not constant, unlike observed 
for Ta. Many of these oxide and oxynitride perovskites tend to typically exhibit $\mathrm{AO}(\mathrm{SrO})$ surface terminations ${ }^{27,42,43}$ rather than $\mathrm{BO}_{2}\left(\mathrm{TaO}_{2}\right)$. As previously discussed, a $\mathrm{Sr} / \mathrm{SrO}_{\mathrm{x}}$ surface initially covered by oxygen adsorbates, would then initially contribute to overall $\mathrm{O}_{2}$ generation. As the potential increases to $1.3 \mathrm{~V}$, the Sr starts to reduce (Fig. 5e) likely associated with the decoupling of the OER intermediates leaving the adsorption site on $\mathrm{Sr}$ vacant ${ }^{20}$ before further adsorption.

However, the degradation in the initial performance of STON (Fig. 1c) suggests this reduction on $\mathrm{Sr}$ is permanent and detrimental. This will be explored more in the next section. When changing the applied potential from 1.3 to $1.5 \mathrm{~V}$, we see no significant change, except an overall further reductive shift albeit, at a reduced magnitude (Fig. 5d insert) within error. This observation could be explained by the earlier discussion regarding the deprotonation of $\mathrm{Sr}$ as the ODS. As the potential increases the majority of the Sr species reduce, followed by $\mathrm{Sr} / \mathrm{SrO} / \mathrm{SrOH}$ leaching. This disorder would also lead to defect Ta $5 \mathrm{~d}$ states near the CBM which will then trap electrons, ${ }^{44}$ leading to chargerecombination. ${ }^{45}$ This, in conjunction with changes seen for $\mathrm{Sr}$ and the generation of $\mathrm{N}$ species in competition with $\mathrm{O}_{2}$ generation, can explain why there is unusually large degradation in the initial photoelectrochemical performance of STON before the material stabilises. 


\section{Modulation Excitation X-ray Absorption Spectroscopy}

In a different and complementary experimental strategy, we performed modulation excitation XAS (ME-XAS) to monitor the real time effects of the chemical modification of the surface using light as the external stimulus. The description of the experiment can be seen in Fig. 6a, where the thin film sample is stimulated by periodic modulation of light (chopped illumination) whilst time resolved XAS measurements are performed and data collected simultaneously during operation conditions at different, fixed potentials (Fig. 6b). Spectral changes induced by external stimuli (light in this case) are often small and/or prone to overshadowing by noise and background signals due to the experimental set-up. ${ }^{46}$ One can improve the signal-to-noise ratio by averaging data sets over several modulation periods. Furthermore, improvements in signal-to-noise ratio can be achieved through analysis of ME-XAS with phase sensitive detection (PSD). ${ }^{47,48,49,50}$ PSD converts time-resolved XAS data into a set of phase $\left(\varphi^{\mathrm{PSD}}\right)$ resolved data akin to a digital lock-in amplifier, enhancing the sensitivity of the ME-XAS experiment to spectral responses that match the frequency of the chopped light stimulus. More information on the PSD approach is included in the Supplementary Discussion. 
Fig. $6 \mathrm{~b}$ shows the chronoamperometry measurements at $1.1,1.3$, and $1.5 \mathrm{~V}$ vs. RHE where the incident light was modulated with 120 -second periods (60 seconds on, 60 seconds off). Fig. $6 \mathrm{~b}$ has been adapted for sake of clarity, where the photocurrent spikes (labelled light ON/OFF), are minimised. At $1.1 \mathrm{~V}$, the photocurrent response is limited, but as the potential is increased further the

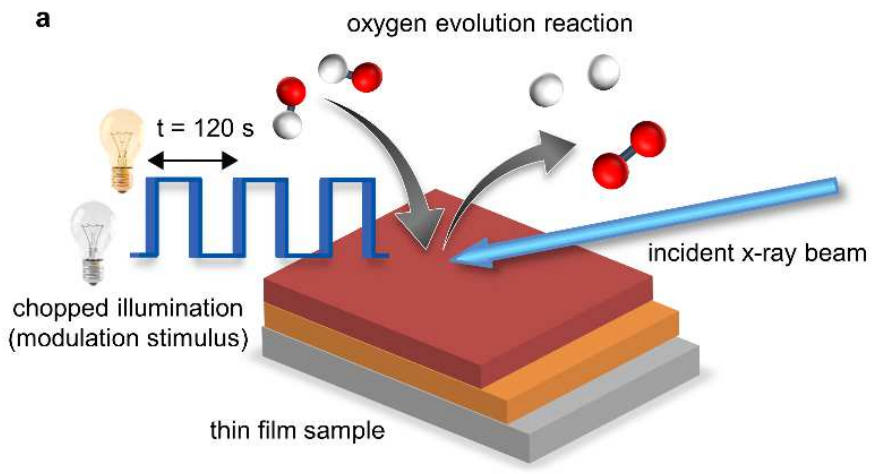

c

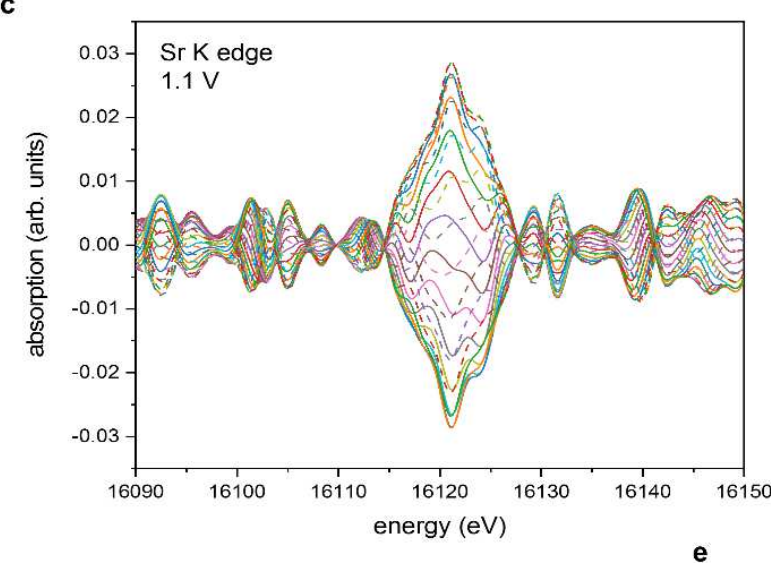

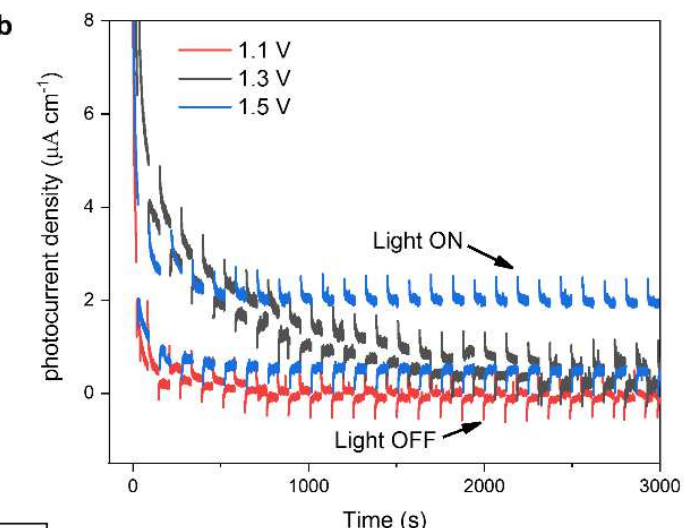

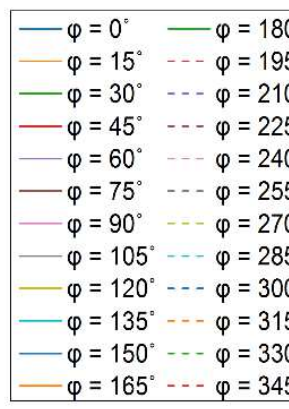

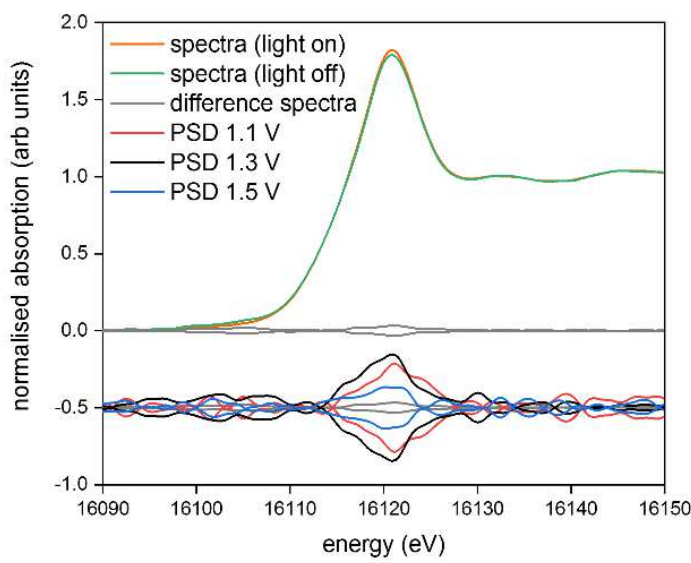


Figure 6. Operando ME-XAS at the STON-liquid interface. (a) schematic of the experiment, (b) chronoamperometry measurements performed at $1.1 \mathrm{~V}, 1.3$ $\mathrm{V}$ and $1.5 \mathrm{~V}$ vs. RHE for 60 minutes under chopped illumination with 60 second periods, (c) phase resolved spectra $\left(\phi^{\mathrm{PSD}}=0^{\circ}-345^{\circ}\right)$ obtained from the modulation experiment measured at $1.1 \mathrm{~V}$ at the $\mathrm{Sr} \mathrm{K}$ edge, (d) phase resolved spectra for Sr K edge measured at 1.1 V, 1.3 V and 1.5 V vs. RHE shown in dark grey, light grey and black respectively, (e) average of spectra recorded under illumination and under dark conditions, with the difference and PSD spectra at each potential.

photocurrent response also increases, as expected. However, at $1.3 \mathrm{~V}$ there is a large increase in the dark current response compared to $1.1 \mathrm{~V}$. This increase degrades over time, before minimising after ca. $3000 \mathrm{~s}$. When the potential is stepped to $1.5 \mathrm{~V}$, there is an initial increase in the dark current response, which stabilises ca. $1 / 3$ faster than at $1.3 \mathrm{~V}$. There is also an overall increase in the stabilised dark current response compared to 1.1 and $1.3 \mathrm{~V}$. These increases in the dark current at $1.3 \mathrm{~V}$ also coincide with the changes seen in the operando XAS data measured at $1.3 \mathrm{~V}$, with the increase of electron density on $\mathrm{Sr}$, resulting in increased overpotentials.

Fig. 6c shows, as an example, the PSD data analysis of data acquired at the $\mathrm{Sr} \mathrm{K}$ edge for STON measured at $1.1 \mathrm{~V}$. The benefit of PSD is the significantly enhanced signal-to-noise ratios, as it contains primarily only the signals from 
the changes due to the external light stimulus. ${ }^{51,52}$ Spurious effects from inactive species, spectators and noise signals are filtered out. We also performed this analysis on the Ta B site (supplementary Fig. 6). However, unlike Sr, the Ta signals never increase above the level of noise. This suggests either two things: 1) Ta is not responsive to the light and not involved in the photo absorption process, a hypothesis which would contradict available literature $\left.{ }^{14,20}{ }^{53} \mathrm{Or}, 2\right)$ the kinetics for Ta are faster than those for $\mathrm{Sr}^{20}$ where Ta evolves $\mathrm{O}_{2}$ at faster rates. Therefore, due to the time resolution of XAS of 0.5 seconds per scan, Ta appears not to change, since only a small fraction of the Ta atoms would be in the excited state. Therefore, we observe changes in electron density on the $\mathrm{Sr}$ sites, as the OER proceeds at much slower rates compared to Ta.

Fig. $6 \mathrm{~d}$ shows the phase resolved spectra for Sr measured at 1.1, 1.3, and $1.5 \mathrm{~V}$ in red, black and blue, respectively. Not only can we see that there are small changes due to stimulus with light, but there is also a potential dependence. The spectra collected at $1.5 \mathrm{~V}$ show decreased sensitivity (smaller differences) due to the modulated light stimulus compared to 1.1 and $1.3 \mathrm{~V}$. Fig. 6e shows an example of averaged spectra collected under illumination and dark conditions at one potential, along with the differential of the two plots shown as a solid grey line and the PSD spectra shown below. Fig. 6e shows that small changes in the intensity of the Sr K-edge occur upon the irradiation of the STON sample with 
light. Upon irradiation with light, photons with sufficient energy are absorbed; generating electrons and electron hole pair charge carriers. The charge carriers are subsequently consumed in the water splitting process or recombine. The promotion of electrons from the valence band to the conduction band will induce small changes in the overall electronic system. This is reflected by the increase in intensity of the Sr K-edge whiteline under illumination (Fig. 6e) . Both the A ( $\mathrm{Sr}$ ) and B (Ta) are active sites for the OER mechanism to proceed on. However, since the OER mechanism (Fig. 5f) proceeds more sluggish on $\mathrm{Sr}$ compared to Ta, as our ME-XAS characterisation suggests, the number of $\mathrm{Sr}$ atoms present in excited state increase as the charges are longer lived and continuously generated under illumination. This is observed as changes in electron density on $\mathrm{Sr}$, reflected in the changes in intensity of the main peak.

Under illumination conditions there is the generation of electron hole pairs on Sr. Electrons are excited from the valence band to the conduction band; the unoccupied antibonding states $\mathrm{Sr} 5 \mathrm{~s}, 4 \mathrm{p}$ and $4 \mathrm{~d}$ (forbidden due to selection rules). We observe an increase in electron density upon occupation of the excited states, reflected by the increase of the white line intensity as well as, the shapes and intensity of the PSD signals. As the potential is increased to $1.5 \mathrm{~V}$, the generated charges under illumination are consumed quicker. Represented by the decrease in the white line and PSD signal intensity. The changes in the 
shape of the PSD signal itself result from the changing occupation of the $5 \mathrm{~s}, 4 \mathrm{p}$ and forbidden $4 \mathrm{~d}$ states. This is reflected by the reduction of Sr with applied potential (occupied 5s orbital) as well as, the change in hybridisation and disorder surrounding $\mathrm{Sr}$ with respect to the proceeding of the OER.

At the higher potentials $(1.5 \mathrm{~V})$ the small changes are reduced in intensity, perhaps, due to the increased rate of the OER, seen by the increase in the photocurrent density (Fig. 6b). The suggested changes to the surface stoichiometry, tensile strain and charge of the surface layer result in a surface reconstruction that would affect the surface binding energies of the intermediate products produced during OER conditions $\left(* \mathrm{OH},{ }^{*} \mathrm{O},{ }^{*} \mathrm{OOH}\right)$. The intermediates that adsorb too weakly or too strongly will reduce the overall kinetics and, increase the overpotential of the OER. ${ }^{29,54}$ At $1.5 \mathrm{~V}$, the applied potential overcomes the increased overpotentials, reflected in the increased photocurrent response. As the kinetics on Sr increase, we are no longer observing changes in electron density, similar for Ta. Since, the kinetics increases the excited state fraction, which is smaller at $1.5 \mathrm{~V}$ (Fig. 6d). Suggesting that the remaining reduced $\mathrm{Sr}$ species that have not leached into the electrolyte, behaves more like Ta, contributing to $\mathrm{O}_{2}$ evolution. Where, the system may not have time to either change as the generated charges are more energetic and consumed in the evolution of $\mathrm{O}_{2}$ at faster rates. 
The reduced intensity of changes shown on $\mathrm{Sr}$ with light modulation at $1.5 \mathrm{~V}$ may also relate to the changes suggested by the static XAS and XPS measurements in relation to the OER. The XAS measurements (Fig. 5e) show that, as the applied potential is further increased from 1.3 to $1.5 \mathrm{~V}, \mathrm{Sr}$ is reduced further but the rate of reduction changes. Deprotonation was shown to be the ODS for STON ${ }^{20}$ Therefore, upon deprotonation, most $\mathrm{Sr}$ species would exist as $\mathrm{SrO}$ which, as previously discussed is partially soluble in the electrolyte to form ionic $\mathrm{Sr}^{2+}$ and $2 \mathrm{OH}$. Sr covered in $\mathrm{OH}$ adsorbates would contribute to $\mathrm{O}_{2}$ generation. However, as the potential increases, the oxidation change on $\mathrm{Sr}$ would change the lattice energy. Therefore, dissolution and leaching of $\mathrm{Sr}^{2+}$ into the electrolyte would be expected. As the reactions proceed, the change of rate in reduction corresponds to the remaining non-reduced $\mathrm{Sr}$ species and increase in overpotentials.

\section{Discussion}

Operando GIXAS was performed at various, constant applied potentials with the sample under illumination. In addition, we used ME-XAS coupled with PSD under chopped illumination to probe the oxynitride photocatalysts using light as external stimulus, checking for reversibility of the system whilst increasing 
signal-to-noise ratios. Our measurements show that the applied potentials and the proceeding of the OER are the driving force behind the detrimental surface evolution of the photocatalyst. The degradation noted for STON in this work are summarised as follows: (a) an increase in electron density on $\mathrm{Sr}$ and its partial dissolution into the alkaline electrolyte (b) apparent Ta enrichment at the surface, with a slight increase in disorder of its local environment resulting in changes in hybridisation with the $\mathrm{N} 2 \mathrm{p}$ and $\mathrm{O} 2 \mathrm{p}$ states. (c) Increase in surface hydrophilicity, evidenced by the increased adsorbed $\mathrm{OH} / \mathrm{O}(\mathrm{OH})$ and $\mathrm{H}_{2} \mathrm{O}$ content. d) Slight loss of $\mathrm{N}$ from the structure (where $\mathrm{N}$ remains chemisorbed as $\mathrm{N}_{2} / \mathrm{NO}_{\mathrm{x}}$ species).

Surface reconstruction under OER conditions has previously been discussed for Sr and Co containing perovskite oxide catalysts ${ }^{55,56,57,58}$ where, the OER proceeds under applied potential together with the lattice-oxygen evolution reaction. ${ }^{55,59}$ This has been shown to have a beneficial effect with respect to the OER due to the formation of an enriched $\mathrm{BOH} / \mathrm{BO}(\mathrm{OH})$ surface, increased hydrophilicity and an increase in catalytic activity. ${ }^{55,60}$

For the oxynitride STON, we also observe surface enrichment of the B cation (Ta) and increased hydrophilicity. However, contrary to oxides, for oxynitrides this superficial $\mathrm{BOH} / \mathrm{BO}(\mathrm{OH})$ enriched surface layer may have complications. 
We observe in the $\mathrm{N} 1 \mathrm{~s}$ XPS spectra that there are $\mathrm{N}_{2} / \mathrm{NO}_{\mathrm{x}}$ chemisorbed species, which are formed in competitive reactions from the surface lattice $\mathrm{N}$ and the OER intermediates. We would like to focus our discussion on the PEC nitrogen evolution reactions at the surface of the oxynitride $\mathrm{SC}$, since the literature on this topic is scarce. Although, this process seems to have important consequences on the oxygen and hydrogen evolution half reactions.

Photocatalytic nitrate formation on Ti dioxide surfaces irradiated by UV or sunlight irradiation under atmospheric $\mathrm{N}_{2}$ and $\mathrm{O}_{2}$ has been discussed by Yuan $e t$ $a l .{ }^{61}$ Where the photogenerated holes on the Ti SC surface catalyse the intermediary $\mathrm{NO}_{\mathrm{x}}$ species according to the following reactions given by equations (1) and (2):

$$
\begin{gathered}
\mathrm{N}_{2}+\mathrm{O}_{2} \rightarrow 2 \mathrm{NO} \\
\mathrm{N}_{2}+2 \mathrm{H}_{2} \mathrm{O}+4 \mathrm{~h}^{+} \rightarrow 4 \mathrm{H}^{+}+2 \mathrm{NO}
\end{gathered}
$$

Dai et $a l^{62}$ recently looked at the nitrogen oxidation reaction (NOR) catalysed by $\mathrm{Fe} / \mathrm{Co}$ spinel oxides in alkaline electrolyte. Where they show that adsorbed $\mathrm{N}_{2}$ is oxidised to intermediate $\mathrm{NO}_{\mathrm{x}}$ species by the adsorbed $\mathrm{OH}$ and the OER intermediate $\mathrm{O}(\mathrm{OOH})$ according to equations (3) and (4): 


$$
\begin{gathered}
\mathrm{N}_{2}+2 \mathrm{OH}^{-} \rightarrow \mathrm{N}^{*}+\mathrm{NO}+\mathrm{H}_{2} \mathrm{O}+2 e^{-} \\
\mathrm{N}^{*}+2 \mathrm{OH}^{-} \rightarrow \mathrm{NO}+\mathrm{H}_{2} \mathrm{O}+2 e^{-}
\end{gathered}
$$

Where the intermediate $\mathrm{NO}_{\mathrm{x}}$ species are oxidised given by equations (5) and (6):

$$
\begin{aligned}
& \mathrm{NO}+2 \mathrm{OH}^{-} \rightarrow \mathrm{NO}_{2}+\mathrm{H}_{2} \mathrm{O}+2 e^{-} \\
& \mathrm{NO}_{2}+2 \mathrm{OH}^{-} \rightarrow \mathrm{NO}_{3}^{-}+\mathrm{H}_{2} \mathrm{O}+e^{-}
\end{aligned}
$$

With the aid of theoretical calculations, the authors note that Co shows increased hybridisation with the $\mathrm{O} 2 \mathrm{p}$ states, which aids in the stabilisation of $\mathrm{OH}^{-}$adsorption. This superficial $\mathrm{CoO}(\mathrm{OH})$ enriched surface layer has a beneficial effect on the OER for the oxides. ${ }^{55,62}$ However, in the presence of surface nitrogen based species, the stabilised $\mathrm{CoO}(\mathrm{OH})$ contribute to both the NOR and OER. ${ }^{62}$

Kato and $\mathrm{Kudo}^{63}$ looked at numerous tantalate oxide photocatalysts, and the authors observed nitrate reduction under UV irradiation in an aqueous environment. Where nitrate forms intermediate nitrite, dinitrogen and ammonia, 
even in the absence of a co-catalyst or reducing agents. The authors proposed the following mechanisms shown in equations (7-10):

$$
\begin{gathered}
\mathrm{NO}_{3}^{-}+2 \mathrm{H}^{+}+2 e_{\mathrm{CB}}^{-} \rightarrow \mathrm{NO}_{2}^{-}+\mathrm{H}_{2} \mathrm{O} \\
\mathrm{NO}_{2}^{-}+7 \mathrm{H}^{+}+6 e_{\mathrm{CB}}^{-} \rightarrow \mathrm{NH}_{3}+2 \mathrm{H}_{2} \mathrm{O} \\
2 \mathrm{NO}_{3}^{-} \rightarrow 2 \mathrm{NO}_{2}^{-}+\mathrm{O}_{2} \\
2 \mathrm{NH}_{3}+6 \mathrm{~h}_{V B}^{+} \rightarrow \mathrm{N}_{2}+6 \mathrm{H}^{+}
\end{gathered}
$$

Where the photogenerated electrons in the $\mathrm{CB}$ reduce the nitrate and nitride species (equations7-8). Alternatively, the nitrate species can undergo photochemical decomposition (equation (9)). The photogenerated holes in the VB can then oxidise ammonia to form dinitrogen (equation (10)), which can form $\mathrm{NO}_{\mathrm{x}}$ species according to equations (1-4).

Wei et al. ${ }^{64}$ recently reported increased photocatalytic activity for nitride reduction on $\mathrm{Ni}_{2} \mathrm{P}$ modified tantalum nitride and tantalum oxynitride $\left(\mathrm{Ta}_{3} \mathrm{~N}_{5}\right.$ and TaON, respectively). Where the photogenerated holes in the VB of the two materials are involved in the water oxidation reaction (equation (12)) and, the photogenerated electrons in the $\mathrm{CB}$ transfer to the $\mathrm{Ni}_{2} \mathrm{P}$ states where they are involved in the photocatalytic reduction of $\mathrm{NO}_{3}{ }^{-}$. For a detailed overview on the 
role of $\mathrm{N}$ in electrocatalysis, the readers are directed to the work by Rosca $e t$ $a l .{ }^{65}$

The photocurrent is proportional to stoichiometric $2: 1 \mathrm{H}_{2} / \mathrm{O}_{2}$ generation, according to the two half reactions of the water splitting process on a SC photocatalyst (equations (11-12)).

$$
\begin{gathered}
4 H^{+}+4 e_{C B}^{-} \rightarrow 2 H_{2} \\
2 H_{2} \mathrm{O} \rightarrow 4 h_{V B}^{+}+O_{2}+4 H^{+}
\end{gathered}
$$

As the potential increases, the forward reactions for $\mathrm{O}_{2}$ generation dominate since the nitrogen content at the surface is limited, compared to the high $\mathrm{OH}^{-}$ content in the alkaline electrolyte. Once the nitrogen species desorbs from the surface, the OER could proceed on the now vacant active site and/or the adsorbed intermediates would no longer contribute to $\mathrm{N}_{2} / \mathrm{NO}_{\mathrm{x}}$ formation in competition. As shown by the increase in photocurrent density as seen in Fig. 1c and $6 \mathrm{~b}$. However, the above-mentioned changes for STON result in a surface reconstruction during the initial stages of operation, reducing its long-term performance. 
As previously discussed, the CB of STON is primarily comprised of Ta $5 \mathrm{~d}$ states. Considering the changes in the disorder, especially the local disorder surrounding the Ta cations, this would result in generation of defect Ta states at/near to the CB minimum or, even mid bandgap defect states. These defect states would trap the photogenerated electrons, leading not only to charge recombination, as previously discussed but also, contribute to the reduction of the generated nitrate species (equations (7-8)) as suggested by the works by Kato and Kudo (2002), Wei et al. (2020) and the XPS analysis (Fig. 3).

The CB minimum and VB maximum of STON incorporate not only the redox potentials of the OER and HER, but also those for $\mathrm{NO}_{3}{ }^{-} / \mathrm{NH}_{4}{ }^{+}(0.88 \mathrm{~V}), \mathrm{NO}_{3}^{-}$/ $\mathrm{HNO}_{2}(0.94 \mathrm{~V})$ and $\mathrm{NO}_{3}{ }^{-} / \mathrm{N}_{2}(1.24 \mathrm{~V})$ at $\mathrm{pH}=0$, vs. NHE. ${ }^{64}$ This implies that all photogenerated electrons consumed in these competing reactions would no longer contribute to the HER at the Pt counter electrode (Fig. 1a), reducing the potential magnitude of the photocurrent density. The photogenerated holes that do not recombine would then primarily contribute to water oxidation (equation (12)).

The surface nitrogen that remains chemisorbed in the form of molecular $\mathrm{N}_{2} / \mathrm{NO}_{\mathrm{x}}$ would likely, a) occupy possible $\mathrm{O}_{2}$ generation sites, effectively blocking the site until it desorbs or, $b$ ) reduce or oxidise to form additional chemisorbed 
$\mathrm{N}_{2} / \mathrm{NO}_{\mathrm{x}}$ species as suggested by XPS. The apparent mechanism is likely then associated with one or more of the previously discussed equations (1-10). Since the electron density surrounding $\mathrm{Ta}$ is conserved, the nitrogen vacancies generated are then likely healed by the OER intermediate adsorbates. ${ }^{29}$ The changing stoichiometry, tensile strain and changes in the charges of the surface layer result in a surface reconstruction that would affect the surface binding energies of the intermediate products produced during OER conditions $(* \mathrm{OH}$, $\left.* \mathrm{O},{ }^{*} \mathrm{OOH}\right)$. The intermediates that adsorb too weakly or too strongly will reduce the overall kinetics and, increase the overpotential of the OER. ${ }^{29,54}$

To conclude, OER and HER remain the dominant reactions, however the initial reconstruction due to Sr dissolution and the following competitive reactions, limit the overall efficiency of STON compared to its initial performance.

\section{Conclusions}

In this work, we employed operando grazing incidence and modulated excitation X-ray absorption spectroscopy to study the effect of several stimuli on STON oxynitride thin films during photoelectrochemical water splitting. A comparison with ex-situ XPS of the samples in their initial and final states is also included. Overall, the XPS analysis of the initial and final state of the 
STON photocatalyst suggests that STON suffers from a surface degradation and reconstruction as evidenced with respect to the decrease in initial photocurrent. The XPS data suggests that STON undergoes a) slight loss of $\mathrm{N}$ from the structure where $\mathrm{N}$ remains bound as $\mathrm{N}_{2} / \mathrm{NO}_{\mathrm{x}}$ species. b) The increase in electron density and segregation of Sr. c) apparent Ta enrichment at the surface due to the changing $\mathrm{O} / \mathrm{N}, \mathrm{d}$ ) Increased hydrophilicity.

Operando GIXAS corroborates the XPS findings, determining that the surface reconstruction occurs when the applied potential increases above $1.3 \mathrm{~V}$ vs. RHE. ME-XAS-PSD measurements. With modulated light as an external stimulus we show that other than the generation of charge carriers, the light has minimal effect on the system, where it was previously shown for $\mathrm{LaTiOxN}_{\mathrm{y}}$ that light and the electrolyte do not contribute to the degradation of the material. ${ }^{31}$ Whereas, in this work, $\mathrm{Sr}$ is an alkali earth element, more soluble at $\mathrm{pH} 13$. That said, we have observed that Ta does not respond to the light on the time scales of the modulation period used in this work (120 s). However, we observe changes in the light response for $\mathrm{Sr}$, with respect to its electronic structure and its evolution as a function of applied potential. Indicating that the kinetics of the OER are much faster on the Ta site than for Sr. However, when the potential is increased to $1.5 \mathrm{~V}$, the rate of the OER on the reduced $\mathrm{Sr}$ sites increase, where $\mathrm{Sr}$ behaves more like a transition metal and less ionic in nature. 
Here we show that the nature of the A cation and the evolution of its electronic and geometric environment at the solid/liquid interface has large impacts on the overall stability and catalytic activity of the material during operation conditions. We also note that nitrogen determines the bulk electronic structure of STON and in turn, the light absorption properties of the photocatalyst. However, at the surface the nitrogen species take on a more apparent antagonistic role, competing with the OER. To improve the performance of semiconductor photocatalysts, it is important to understand on a fundamental level what is occurring at the photocatalyst surface during operation. Future work plans to explore in operando, the effect of applied potential and the synergistic effects first row transition metal based co-catalysts have on the performance and stability of the oxynitride photocatalyst templates, where it has been shown that passivation layers and co-catalysts can prevent/minimise the detrimental surface reconstruction.

\section{Methods}

\section{Thin Film Deposition}

Three sets of films used in this work were grown using pulsed laser deposition (PLD). A KrF excimer laser (Lambda Physik LPX 300, 30 ns pulse width, $\lambda=$ 
$248 \mathrm{~nm}$ ) was used to ablate a target of $\mathrm{Sr}_{2} \mathrm{Ta}_{2} \mathrm{O}_{7}$ fabricated in our laboratory.

The target to substrate distance was set at $5 \mathrm{~cm}$. The laser was focused on a spot of $1.1 \mathrm{~mm}^{2}$ with a laser fluence of ca. $3 \mathrm{~J} \mathrm{~cm}^{-2}$ and laser repetition rate of $10 \mathrm{~Hz}$. Commercially available (001)-oriented $\mathrm{MgO}$ was used as a substrate $(10 \mathrm{x} 10 \mathrm{x}$ $0.5 \mathrm{~mm}$ ). Platinum paste was applied between the substrates and heating stage to provide good thermal conductivity. The substrate temperature of $750^{\circ} \mathrm{C}$ was measured via a pyrometer. $\mathrm{N}_{2}$ background partial pressure of $8.0 \times 10^{-4} \mathrm{mbar}$ was set via a gas inlet line to the vacuum chamber. $\mathrm{NH}_{3}$ gas was injected through a nozzle near the laser spot at the target. The titanium nitride current collector layer was grown in situ, prior to the deposition of the oxynitride film, by conventional PLD using a commercially available TiN target under vacuum with a base pressure of ca. $5 \times 10^{-6}$ mbar. The substrate to target distance, substrate temperature, laser repetition and fluence were the same as above.

\section{Photoelectrochemical Characterisation}

Photoelectrochemical (PEC) measurements were performed using a threeelectrode configuration in the operando reactor cell described in Fig. 3. The working and counter electrode were the STON thin films and Pt wire respectively. $\mathrm{A} \mathrm{KCl}$ saturated $\mathrm{Ag} / \mathrm{AgCl}$ electrode was used as the reference. $\mathrm{An}$ aqueous solution of 0.5 molar $\mathrm{NaOH}(\mathrm{pH}=13)$ was used as an electrolyte. For the electrical contact of the STON films a wire was adhered to the TiN current 
collector to apply the electrical contact to the potentiostat (Metrohm Autolab). The electrically connected area was then insulated with epoxy and the sample then immersed into the electrolyte in the cell. The samples were illuminated with a $405 \mathrm{~nm}$ laser diode (Laser2000) which has a $5 \mathrm{~mW}$ power output and spot size of ca. $0.0308 \mathrm{~cm}^{2}$. The corresponding light intensity is ca. $130 \mathrm{~mW} \mathrm{~cm}$. To measure the dark and light current the laser diode was modulated for 60 second periods ( 60 seconds on, 60 seconds off). The potentiodynamic measurements were performed at a scan rate of $10 \mathrm{mv} \mathrm{s}^{-1}$ under chopped illumination with 4 second periods.

\section{Chemical Composition}

Rutherford Backscattering (RBS) and Elastic Recoil Detection Analysis (ERDA) were employed to determine the chemical compositions of the STON thin films. RBS provides the metal ratios and oxygen content whereas, ERDA provides nitrogen-to-oxygen ratio. The RBS measurements were performed using a $2 \mathrm{MeV}$ He beam and a silicon PIN diode detector. ERDA was carried out with a $13 \mathrm{MeV}{ }^{127} \mathrm{I}$ beam and the combination of a time-of-flight spectrometer with a gas ionisation detector. RBS data was analysed using RUMP. ${ }^{66}$ 


\section{Crystalline Properties}

XRD measurements were performed using a Seifert X-ray Diffractometer with characteristic $\mathrm{Cu} \mathrm{K} \alpha$ radiation $0.154 \mathrm{~nm}$. Theta-2theta scans were performed to determine the out-of-plane orientations of the films.

\section{X-ray Photoelectron Spectroscopy}

The XPS measurements were performed at the SX-ARPES endstation ${ }^{67}$ of the Advanced Resonant Spectroscopies (ADRESS) beamline ${ }^{68}$ situated at the Swiss Light Source, Paul Scherrer Institute, Switzerland. The photon flux was ca. $10^{13}$ photons s$~^{-1}$ and focused into a spot size of $30 \times 75 \mu \mathrm{m}^{2}$ on the sample surface at an X-ray grazing incidence angle of $20^{\circ}$. The energy resolution was set to 50 $\mathrm{meV}$ and the sample temperature was kept at $298 \mathrm{~K}$. Spectra were calibrated using the $\mathrm{C} 1 \mathrm{~s}$ signal due to adventitious carbon situated at $284.8 \mathrm{eV}$.

\section{X-ray Absorption Spectroscopy}

The time resolved XAS were measured using the quickXAS method ${ }^{50}$ at the SuperXAS beamline at the SLS, Paul Scherrer Institut in Switzerland. The polychromatic beam of the 2.9 Tesla superbend was collimated by a Si-coated collimating mirror and subsequently monochromatised by a Si (111) channelcut crystal of the quickXAS monochromator, which oscillated at $1 \mathrm{~Hz}$ frequency with an acquisition time of 500 milliseconds per spectrum. Energy calibration 
was performed using $\mathrm{Pb}\left(\mathrm{L}_{1}\right.$ edge $\left.\mathrm{E}_{0}=15861 \mathrm{eV}\right)$ and $\mathrm{Zn}\left(\mathrm{K}\right.$ edge $\mathrm{E}_{0}=9659$ $\mathrm{eV})$ thin foils for the $\mathrm{Sr} \mathrm{K}(\mathrm{E} 0=16105 \mathrm{eV})$ and $\mathrm{Ta}_{3}(\mathrm{E} 0=9881 \mathrm{eV})$ edges, respectively. A focused beam was used with a spot size of $100 \times 100 \mu \mathrm{m}^{2}$. The samples were fixed into the cell and the cell was mounted in grazing incidence to the incoming X-ray beam, with an incident angle between 0-1 degrees. Data analysis was performed using the ProQEXAFS, ${ }^{69}$ Athena $^{70}$ and $\operatorname{Larch}^{71}$ software packages.

\section{Acknowledgements}

The authors would like to thank the Paul Scherrer Institut for financial support through a CROSS grant and the Swiss Light Source for the provision of beamtime at the SuperXAS beamline. TJS thanks Innosuisse and the Swiss Competence Center for Energy Research (SCCER) Heat \& Electricity Storage for financial support.

\section{Author Contributions}

Conceptualisation, C.L., A.C., O.S., M.N., and D.P.; Investigation, C.L., A.C., Z.P., O.S., V.S., M.D.; Formal Analysis, C.L., A.C., Z.P., V.S.; M.D.; Writing Original Draft, C.L. and D.P.; Writing - Review \& Editing, C.L., A.C., Z.P., O.S., M.N., V.S., M.D., T.S., D.P and T.L.; Funding Acquisition, D.P., M.N., T.S., T.L.; Supervision, V.S., M.N., D.P., and T.L. 


\section{Data Availability}

The data used in this study is available from the corresponding author upon reasonable requests.

\section{References}

1. Tian Y, Zhao CY. A review of solar collectors and thermal energy storage in solar thermal applications. Applied Energy 104, 538-553 (2013).

2. Parida B, Iniyan S, Goic R. A review of solar photovoltaic technologies. Renewable and Sustainable Energy Reviews 15, 1625-1636 (2011).

3. Montoya JH, Seitz LC, Chakthranont P, Vojvodic A, Jaramillo TF, Norskov JK. Materials for solar fuels and chemicals. Nat Mater 16, 70-81 (2016).

4. Hisatomi T, Domen K. Reaction systems for solar hydrogen production via water splitting with particulate semiconductor photocatalysts. Nature Catalysis 2, 387-399 (2019).

5. Kim JH, Hansora D, Sharma P, Jang JW, Lee JS. Toward practical solar hydrogen production an artificial photosynthetic leaf-to-farm challenge. Chem Soc Rev 48, 1908-1971 (2019).

6. Pinaud BA, et al. Technical and economic feasibility of centralized facilities for solar hydrogen production via photocatalysis and photoelectrochemistry. Energy \& Environmental Science 6, (2013).

7. Hisatomi T, Kubota J, Domen K. Recent advances in semiconductors for photocatalytic and photoelectrochemical water splitting. Chem Soc Rev 43, 7520-7535 (2014).

8. Takata T, Pan C, Domen K. Recent progress in oxynitride photocatalysts for visible-lightdriven water splitting. Sci Technol Adv Mater 16, 033506 (2015). 
9. Takata T, Domen K. Development of non-oxide semiconductors as light harvesting materials in photocatalytic and photoelectrochemical water splitting. Dalton Trans 46, 10529-10544 (2017).

10. Seo J, et al. Photoelectrochemical Water Splitting on Particulate ANbO2N $(A=B a, S r)$ Photoanodes Prepared from Perovskite-Type ANbO3. Chemistry of Materials 28, 6869-6876 (2016).

11. Maeda K, Domen K. Oxynitride materials for solar water splitting. MRS Bulletin 36, 25-31 (2011).

12. Moriya Y, Takata T, Domen K. Recent progress in the development of (oxy)nitride photocatalysts for water splitting under visible-light irradiation. Coordination Chemistry Reviews 257, 1957-1969 (2013).

13. Navarro Yerga RM, Alvarez Galvan MC, del Valle F, Villoria de la Mano JA, Fierro JL. Water splitting on semiconductor catalysts under visible-light irradiation. ChemSusChem 2, 471-485 (2009).

14. Zhong Y, et al. Enhanced Water-Splitting Performance of Perovskite SrTaO2N Photoanode Film through Ameliorating Interparticle Charge Transport. Advanced Functional Materials 26, 7156-7163 (2016).

15. Haydous F, et al. Oxynitride Thin Films versus Particle-Based Photoanodes: A Comparative Study for Photoelectrochemical Solar Water Splitting. ACS Applied Energy Materials 2, 754763 (2019).

16. Wang Y, Wei S, Xu X. SrTaO2N-CaTaO2N solid solutions as efficient visible light active photocatalysts for water oxidation and reduction. Applied Catalysis B: Environmental 263 (2020).

17. Kawashima K, et al. Perovskite Sr1-xBaxW1-yTay(O,N)3: synthesis by thermal ammonolysis and photocatalytic oxygen evolution under visible light. Materials for Renewable and Sustainable Energy 6, (2017).

18. Pichler $\mathrm{M}$, et al. TiN-buffered substrates for photoelectrochemical measurements of oxynitride thin films. Applied Surface Science 369, 67-75 (2016).

19. Pichler M, et al. LaTiOxNy Thin Film Model Systems for Photocatalytic Water Splitting: Physicochemical Evolution of the Solid-Liquid Interface and the Role of the Crystallographic Orientation. Advanced Functional Materials 27, (2017).

20. Ouhbi H, Aschauer U. Water oxidation chemistry of oxynitrides and oxides: Comparing NaTaO3 and SrTaO2N. Surface Science 677, 258-263 (2018). 
21. Wang F, et al. Recent advances in transition-metal dichalcogenide based nanomaterials for water splitting. Nanoscale 7, 19764-19788 (2015).

22. Lide DR. CRC Handbook of Chemistry and Physics, 89th Edition. Taylor \& Francis (2008).

23. Opitz AK, et al. The Chemical Evolution of the La0.6Sr0.4CoO3-delta Surface Under SOFC Operating Conditions and Its Implications for Electrochemical Oxygen Exchange Activity. Top Catal 61, 2129-2141 (2018).

24. Koo B, Kim K, Kim JK, Kwon H, Han JW, Jung W. Sr Segregation in Perovskite Oxides: Why It Happens and How It Exists. Joule 2, 1476-1499 (2018).

25. Pramana SS, et al. Crystal structure and surface characteristics of Sr-doped GdBaCo2O6- $\delta$ double perovskites: oxygen evolution reaction and conductivity. Journal of Materials Chemistry A 6, 5335-5345 (2018).

26. Mullica DF, Lok CK, Perkins HO, Young VV. X-ray photoelectron final-state screening in La(OH)3: A multiplet structural analysis. Phys Rev B Condens Matter 31, 4039-4042 (1985).

27. Staykov A, Tellez H, Druce J, Wu J, Ishihara T, Kilner J. Electronic properties and surface reactivity of SrO-terminated $\mathrm{SrTiO}_{3}$ and $\mathrm{SrO}$-terminated iron-doped SrTiO3. Sci Technol Adv Mater 19, 221-230 (2018).

28. Robin A. Corrosion behaviour of tantalum in sodium hydroxide solutions. Journal of Applied Electrochemistry 33, 37-42 (2003).

29. Ouhbi H, Aschauer U. Nitrogen Loss and Oxygen Evolution Reaction Activity of Perovskite Oxynitrides. Acs Mater Lett 1, 52-57 (2019).

30. Lawley C, et al. Momentum-resolved electronic structure of LaTiOxNy photocatalysts by resonant soft-X-ray ARPES. In preparation.

31. Lawley C, et al. Examining the surface evolution of LaTiOxNy an oxynitride solar water splitting photocatalyst. Nature Communications 11, 1178 (2020).

32. Pichler $M$, et al. Determination of Conduction and Valence Band Electronic Structure of LaTiOx Ny Thin Film. ChemSusChem 10, 2099-2106 (2017).

33. Aguiar R, Logvinovich D, Weidenkaff A, Rachel A, Reller A, Ebbinghaus SG. The vast colour spectrum of ternary metal oxynitride pigments. Dyes and Pigments 76, 70-75 (2008).

34. Paek S-M, Kim Y-I. Ta L3-edge XANES study of perovskite oxynitrides ATaO2N ( $\mathrm{A}=\mathrm{Ca}, \mathrm{Sr}, \mathrm{Ba})$. Journal of Alloys and Compounds 587, 251-254 (2014). 
35. Ibidunni AO, Masaitis RL, Opila RL, Davenport AJ, Isaacs HS, Taylor JA. Characterization of the Oxidation of Tantalum Nitride. Surface and Interface Analysis 20, 559-564 (1993).

36. Wu TS, et al. Correlation between oxygen vacancies and magnetism in Mn-doped Y2O3 nanocrystals investigated by defect engineering techniques. Applied Physics Letters 101, (2012).

37. Cheng X, Kim BJ, Fabbri E, Schmidt TJ. Co/Fe Oxyhydroxides Supported on Perovskite Oxides as Oxygen Evolution Reaction Catalyst Systems. ACS Appl Mater Interfaces 11, 34787-34795 (2019).

38. Kasatikov S, Filatova E, Sakhonenkov S, Konashuk A, Makarova A. Relationship between Ta Oxidation State and Its Local Atomic Coordination Symmetry in a Wide Range of Oxygen Nonstoichiometry Extent of TaOx. The Journal of Physical Chemistry C 123, 6849-6860 (2019).

39. Mahmood A, et al. Cation effect on electronic, optical and thermoelectric properties of perovskite oxynitrides: Density functional theory. Materials Science in Semiconductor Processing 107, (2020).

40. Greegor RB, Pingitore NE, Jr., Lytle FW. Strontianite in coral skeletal aragonite. Science 275, 1452-1454 (1997).

41. Farges F. Coordination of Ti in crystalline and glassy fresnoites: A high-resolution XANES spectroscopy study at the Ti K-edge. J Non-Cryst Solids 204, 53-64 (1996).

42. Staykov A, Fukumori S, Yoshizawa K, Sato K, Ishihara T, Kilner J. Interaction of SrOterminated $\mathrm{SrTiO} 3$ surface with oxygen, carbon dioxide, and water. Journal of Materials Chemistry A 6, 22662-22672 (2018).

43. Druce J, et al. Surface termination and subsurface restructuring of perovskite-based solid oxide electrode materials. Energy Environ Sci 7, 3593-3599 (2014).

44. Lee J, Lu WD, Kioupakis E. Electronic and optical properties of oxygen vacancies in amorphous Ta2O5 from first principles. Nanoscale 9, 1120-1127 (2017).

45. Wen $\mathrm{X}$, et al. Defect trapping states and charge carrier recombination in organic-inorganic halide perovskites. Journal of Materials Chemistry C 4, 793-800 (2016).

46. Abe $\mathrm{H}$, et al. Improving the quality of XAFS data. J Synchrotron Radiat 25, 972-980 (2018).

47. Ferri D, Newton MA, Nachtegaal M. Modulation Excitation X-Ray Absorption Spectroscopy to Probe Surface Species on Heterogeneous Catalysts. Topics in Catalysis 54, 1070-1078 (2011). 
48. Chiarello GL, Ferri D. Modulated excitation extended X-ray absorption fine structure spectroscopy. Phys Chem Chem Phys 17, 10579-10591 (2015).

49. König CFJ, van Bokhoven JA, Schildhauer TJ, Nachtegaal M. Quantitative Analysis of Modulated Excitation X-ray Absorption Spectra: Enhanced Precision of EXAFS Fitting. The Journal of Physical Chemistry C 116, 19857-19866 (2012).

50. Nachtegaal M, Müller O, König C, Frahm R. QEXAFS: Techniques and Scientific Applications for Time-Resolved XAS. In: X-Ray Absorption and X-Ray Emission Spectroscopy (ed^(eds Bokhoven JAV, Lamberti C). John Wiley \& Sons (2016).

51. Baurecht D, Fringeli UP. Quantitative modulated excitation Fourier transform infrared spectroscopy. Review of Scientific Instruments 72, 3782-3792 (2001).

52. Urakawa A, Bürgi T, Baiker A. Sensitivity enhancement and dynamic behavior analysis by modulation excitation spectroscopy: Principle and application in heterogeneous catalysis. Chemical Engineering Science 63, 4902-4909 (2008).

53. Zhang P, Zhang J, Gong J. Tantalum-based semiconductors for solar water splitting. Chem Soc Rev 43, 4395-4422 (2014).

54. Fabbri E, Habereder A, Waltar K, Kötz R, Schmidt TJ. Developments and perspectives of oxide-based catalysts for the oxygen evolution reaction. Catal Sci Technol 4, 3800-3821 (2014).

55. Fabbri E, et al. Dynamic surface self-reconstruction is the key of highly active perovskite nano-electrocatalysts for water splitting. Nat Mater 16, 925-931 (2017).

56. Deng X, Tüysüz H. Cobalt-Oxide-Based Materials as Water Oxidation Catalyst: Recent Progress and Challenges. ACS Catalysis 4, 3701-3714 (2014).

57. May KJ, et al. Influence of Oxygen Evolution during Water Oxidation on the Surface of Perovskite Oxide Catalysts. The Journal of Physical Chemistry Letters 3, 3264-3270 (2012).

58. Das T, Nicholas JD, Qi Y. Polaron size and shape effects on oxygen vacancy interactions in lanthanum strontium ferrite. Journal of Materials Chemistry A 5, 25031-25043 (2017).

59. Fabbri E, Schmidt TJ. Oxygen Evolution Reaction-The Enigma in Water Electrolysis. ACS Catalysis 8, 9765-9774 (2018).

60. Sun X, Wu F, Liu G, Xu X. Enabling efficient visible light photocatalytic water splitting over SrTaO2N by incorporating Sr in its B site. Journal of Materials Chemistry A 6, 20760-20768 (2018). 
61. Yuan SJ, Chen JJ, Lin ZQ, Li WW, Sheng GP, Yu HQ. Nitrate formation from atmospheric nitrogen and oxygen photocatalysed by nano-sized titanium dioxide. Nat Commun 4, 2249 (2013).

62. Dai C, Sun Y, Chen G, Fisher AC, Xu ZJ. Electrochemical Oxidation of Nitrogen towards Direct Nitrate Production on Spinel Oxides. Angew Chem Int Ed Engl 59, $9418-9422$ (2020).

63. Kato $\mathrm{H}$, Kudo A. Photocatalytic reduction of nitrate ions over tantalate photocatalysts. Physical Chemistry Chemical Physics 4, 2833-2838 (2002).

64. Wei L, Adamson MAS, Vela J. Ni2P-Modified Ta3N5 and TaON for Photocatalytic Nitrate Reduction. Chemnanomat 6, 1179-1185 (2020).

65. Rosca V, Duca M, de Groot MT, Koper MT. Nitrogen cycle electrocatalysis. Chem Rev 109, 2209-2244 (2009).

66. Doolittle LR. A Semiautomatic Algorithm for Rutherford Backscattering Analysis. Nucl Instrum Meth B 15, 227-231 (1986).

67. Strocov VN, et al. Soft-X-ray ARPES facility at the ADRESS beamline of the SLS: concepts, technical realisation and scientific applications. J Synchrotron Radiat 21, 32-44 (2014).

68. Strocov VN, et al. High-resolution soft X-ray beamline ADRESS at the Swiss Light Source for resonant inelastic X-ray scattering and angle-resolved photoelectron spectroscopies. J Synchrotron Radiat 17, 631-643 (2010).

69. Clark AH, Imbao J, Frahm R, Nachtegaal M. ProQEXAFS: a highly optimized parallelized rapid processing software for QEXAFS data. Journal of Synchrotron Radiation 27, 551-557 (2020).

70. Ravel B, Newville M. ATHENA,ARTEMIS,HEPHAESTUS: data analysis for X-ray absorption spectroscopy usingIFEFFIT. Journal of Synchrotron Radiation 12, 537-541 (2005).

71. Newville M. Larch: An Analysis Package for XAFS and Related Spectroscopies. Journal of Physics: Conference Series 430, (2013). 


\section{Figure legends}

\section{Figure 1}

Figure 1. Photoelectrochemical characterisation (a) Energy diagram for photocatalytic water splitting, (b) experimental schematic of the photoelectrochemical three electrode cell. The working, counter and reference electrodes are the oxynitride thin film, platinum wire and $\mathrm{Ag} / \mathrm{AgCl}$ respectively, (c) photocurrent densities for STON for the first three potentiodynamic measurements.

\section{Figure 2}

Figure 2. XRD pattern of the epitaxially grown STON film. The $\theta / 2 \theta$ scan is shown in black, with the angular position of the (001) reflexes of $\mathrm{SrTaO}_{2} \mathrm{~N}$ shown in blue for reference. The inset shows the crystal structure of $\mathrm{SrTaO}_{2} \mathrm{~N}$.

\section{Figure 3}

Figure 3. STON thin film XPS spectra before and after PEC. (a) Sr 3d spectra, (b) Sr 3p spectra, (c) Sr 4s, Sr 4p and Ta 4f spectra, (d) Ta 4d spectra, (e) Ta $4 p$ and N 1s spectra, (f) O 1s spectra. Before and after PEC shown in blue and red, respectively. Reported binding energies for the associated chemisorbed $\mathrm{N}$ and $\mathrm{O}$ species are included in parts (e-f) to clarify the convolution of the $\mathrm{N} 1 \mathrm{~s}$ and $\mathrm{Ta} 4 \mathrm{p} 3 / 2$ and the shoulder of the $\mathrm{O}$ 1s spectra, respectively. References are included in the supplementary. 


\section{Figure 4}

Figure 4. Operando reactor cell for surface sensitive GIXAS measurements.

(a) trimetric view of the cell and GIXAS geometry used during measurements, where the grazing incident X-rays enter through a Mylar foil covered side window, (b) cross sectional internal view. Where, PIPS denotes a passivated implanted planar silicon detector.

\section{Figure 5}

Figure 5. Operando GIXAS at the STON-liquid interface. (a) Reference spectra for $\mathrm{Ta}^{5+}$ oxide and oxynitride powders, (b) operando measurements for STON thin film during the chronoamperometry (1 hour) measurements, with stepped applied potential. The error bars included in the magnified insert correspond to $\pm 0.1 \mathrm{eV}$, (c) graphic representation for the suggested changes occurring to the band structure of the surface layers of the STON photocatalyst. $\mathrm{VB}, \mathrm{CB}$ and $\mathrm{E}_{\mathrm{g}}$ denote the valence band, conduction band and band gap, respectively, (d) reference spectra for $\mathrm{Sr}$ containing powders, (e) operando measurements for STON thin film during the chronoamperometry (1 hour) measurements, with stepped applied potential. The error bars included in the magnified insert correspond to $\pm 0.1 \mathrm{eV}$, (f) suggested changes with respect to Sr after PEC. 


\section{Figure 6}

Figure 6. Operando ME-XAS at the STON-liquid interface. (a) schematic of the experiment, (b) chronoamperometry measurements performed at $1.1 \mathrm{~V}, 1.3$ $\mathrm{V}$ and $1.5 \mathrm{~V}$ vs. RHE for 60 minutes under chopped illumination with 60 second periods, (c) phase resolved spectra $\left(\phi^{\mathrm{PSD}}=0^{\circ}-345^{\circ}\right)$ obtained from the modulation experiment measured at $1.1 \mathrm{~V}$ at the $\mathrm{Sr} \mathrm{K}$ edge, (d) phase resolved spectra for Sr K edge measured at 1.1 V, 1.3 V and 1.5 V vs. RHE shown in dark grey, light grey and black respectively, (e) average of spectra recorded under illumination and under dark conditions, with the difference and PSD spectra at each potential. 
Figures

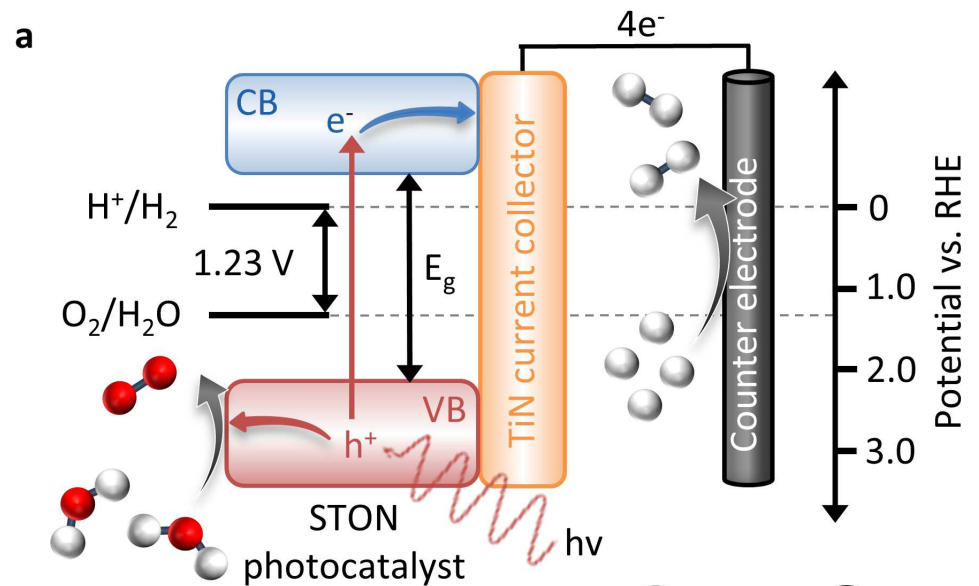

b

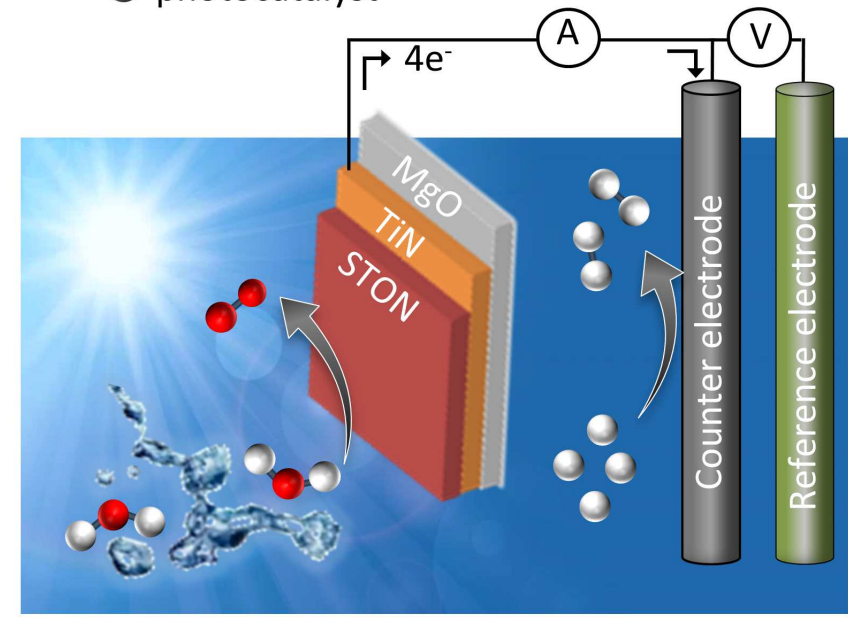

c

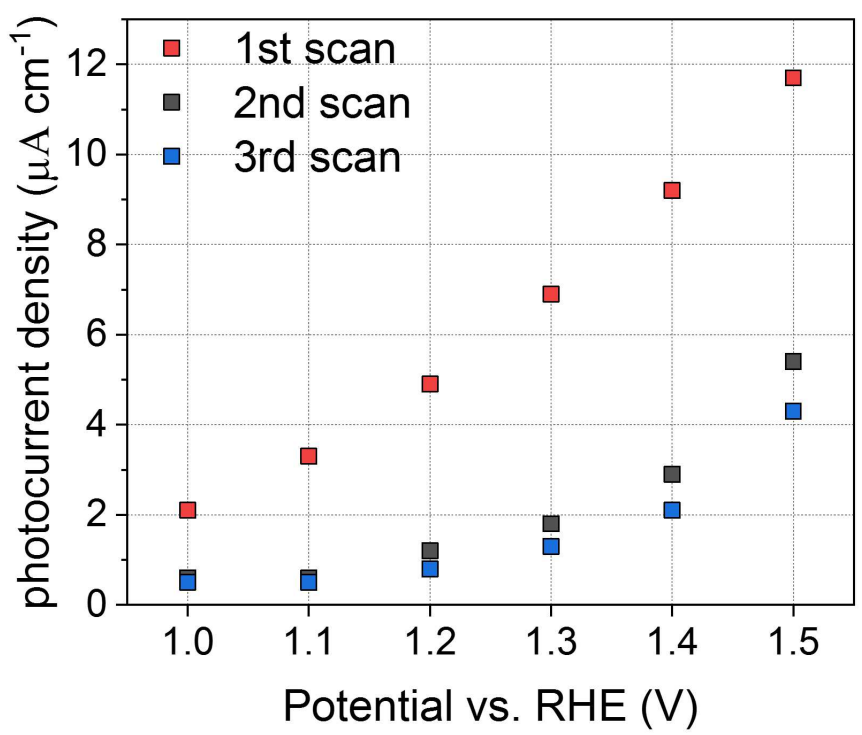

Figure 1

Photoelectrochemical characterisation (a) Energy diagram for photocatalytic water splitting, (b) experimental schematic of the photoelectrochemical three electrode cell. The working, counter and 
reference electrodes are the oxynitride thin film, platinum wire and $\mathrm{Ag} / \mathrm{AgCl}$ respectively, (c) photocurrent densities for STON for the first three potentiodynamic measurements.

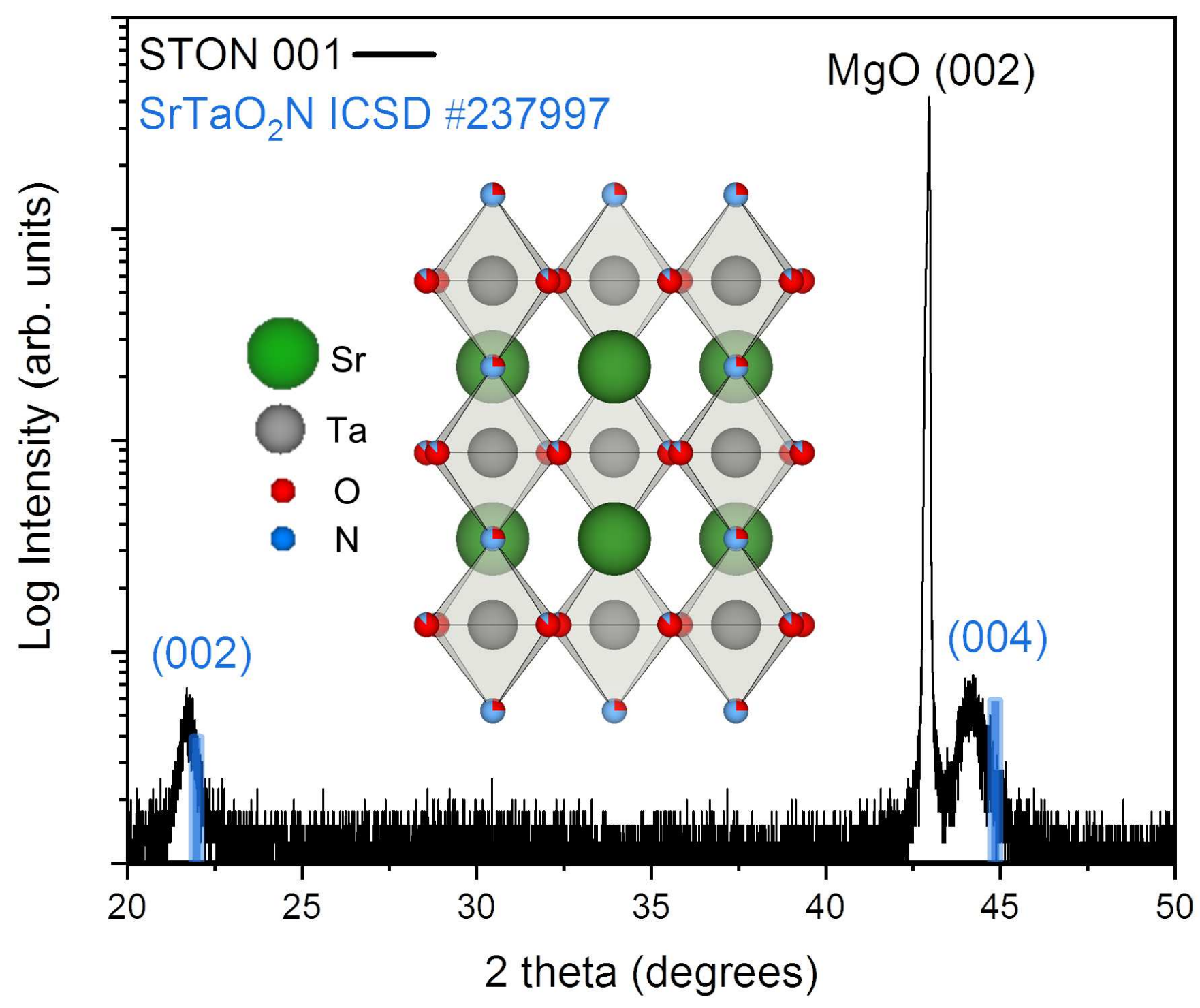

Figure 2

XRD pattern of the epitaxially grown STON film. The $\theta / 2 \theta$ scan is shown in black, with the angular position of the (0OI) reflexes of $\mathrm{SrTaO} 2 \mathrm{~N}$ shown in blue for reference. The inset shows the crystal structure of $\mathrm{SrTaO} 2 \mathrm{~N}$. 
a

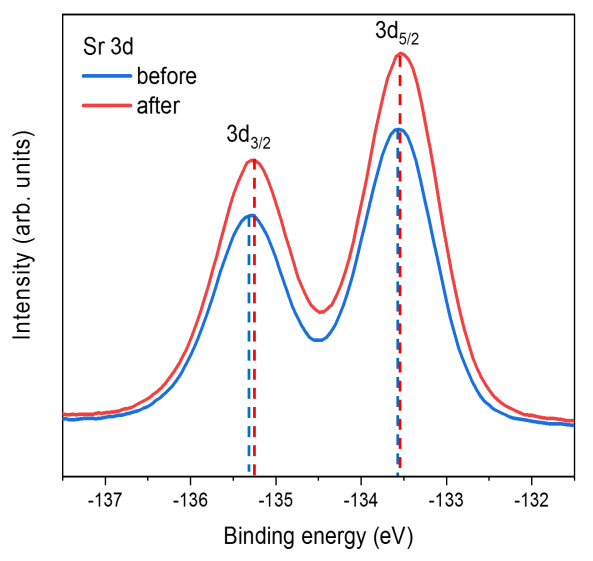

d

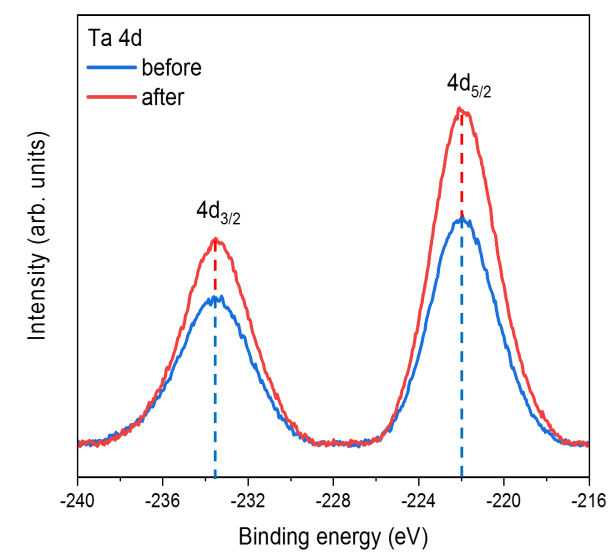

b

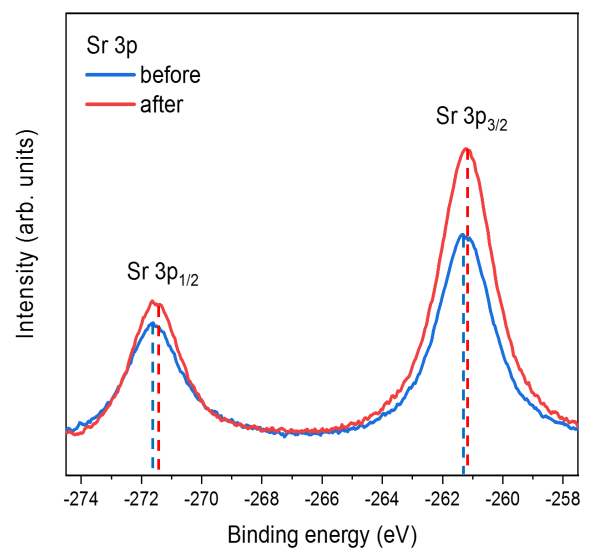

e

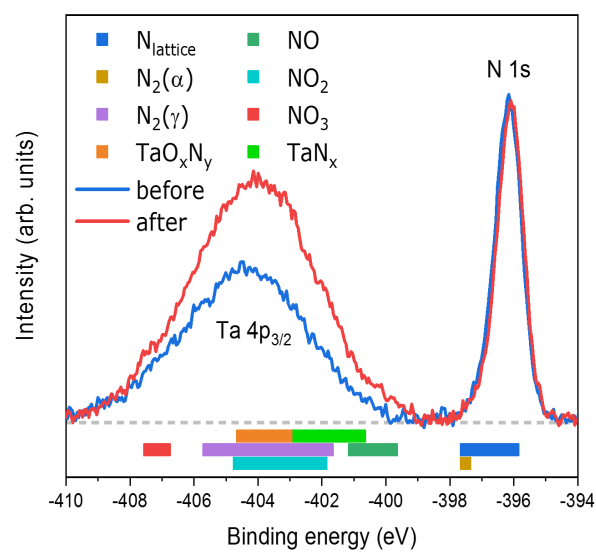

C

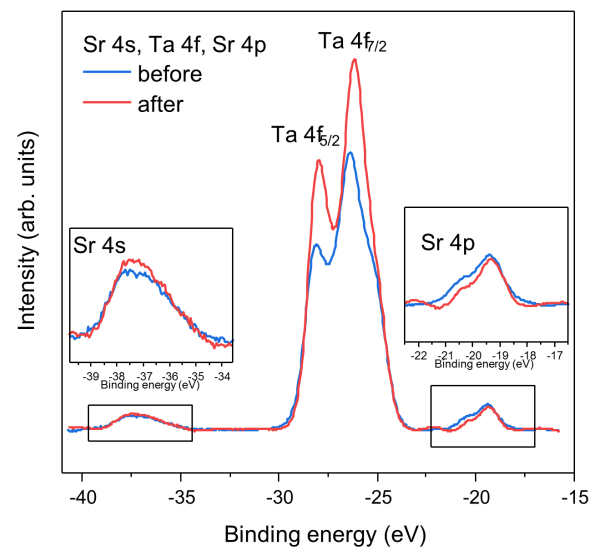

f

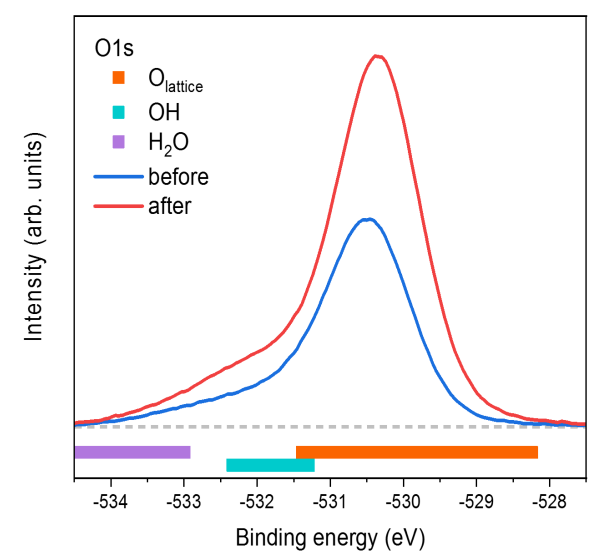

Figure 3

STON thin film XPS spectra before and after PEC. (a) Sr 3d spectra, (b) Sr 3p spectra, (c) Sr 4s, Sr 4p and Ta $4 \mathrm{f}$ spectra, (d) Ta 4d spectra, (e) Ta $4 p$ and N 1s spectra, (f) 01 s spectra. Before and after PEC shown in blue and red, respectively. Reported binding energies for the associated chemisorbed $\mathrm{N}$ and $\mathrm{O}$ species are included in parts (e-f) to clarify the convolution of the $\mathrm{N} 1 \mathrm{~s}$ and Ta $4 \mathrm{p} 3 / 2$ and the shoulder of the $01 \mathrm{~s}$ spectra, respectively. References are included in the supplementary. 
a

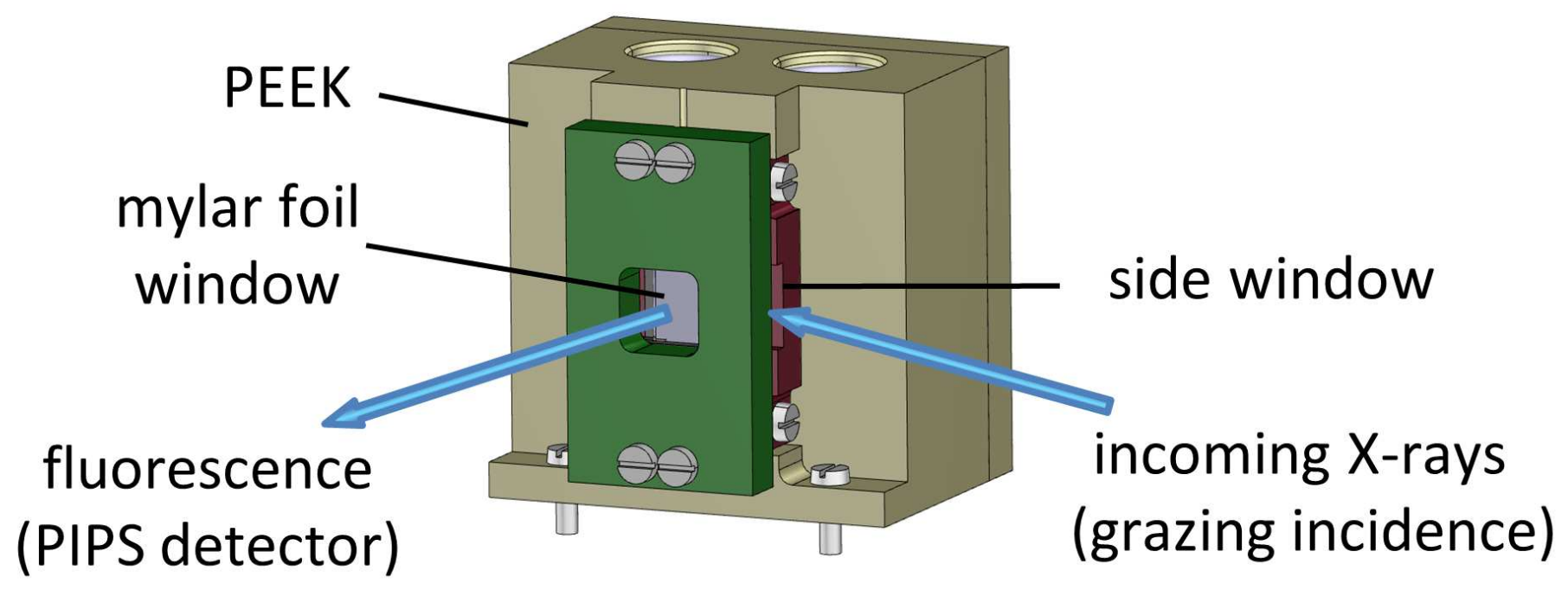

b

\section{Electrode holders}

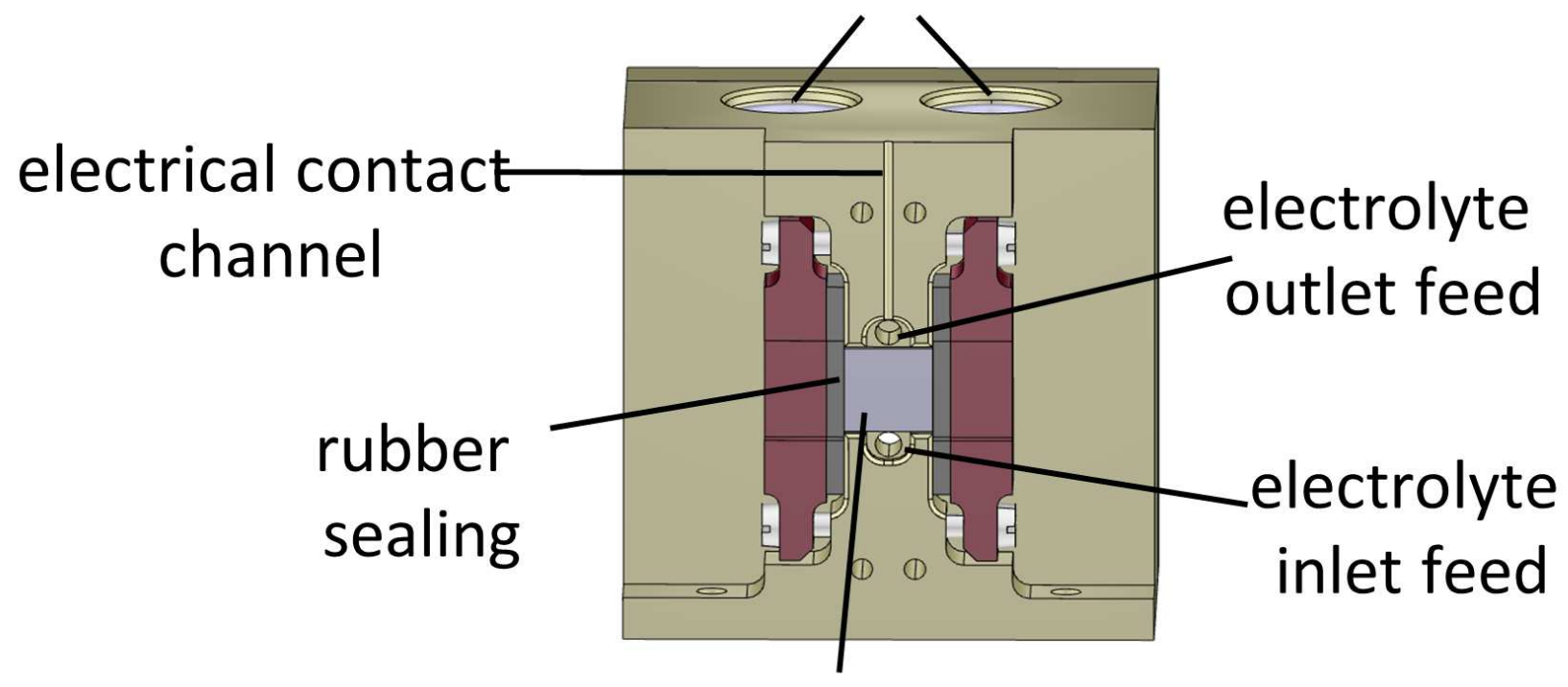

thin film sample

Figure 4

Operando reactor cell for surface sensitive GIXAS measurements. (a) trimetric view of the cell and GIXAS geometry used during measurements, where the grazing incident X-rays enter through a Mylar foil covered side window, (b) cross sectional internal view. Where, PIPS denotes a passivated implanted planar silicon detector. 
a
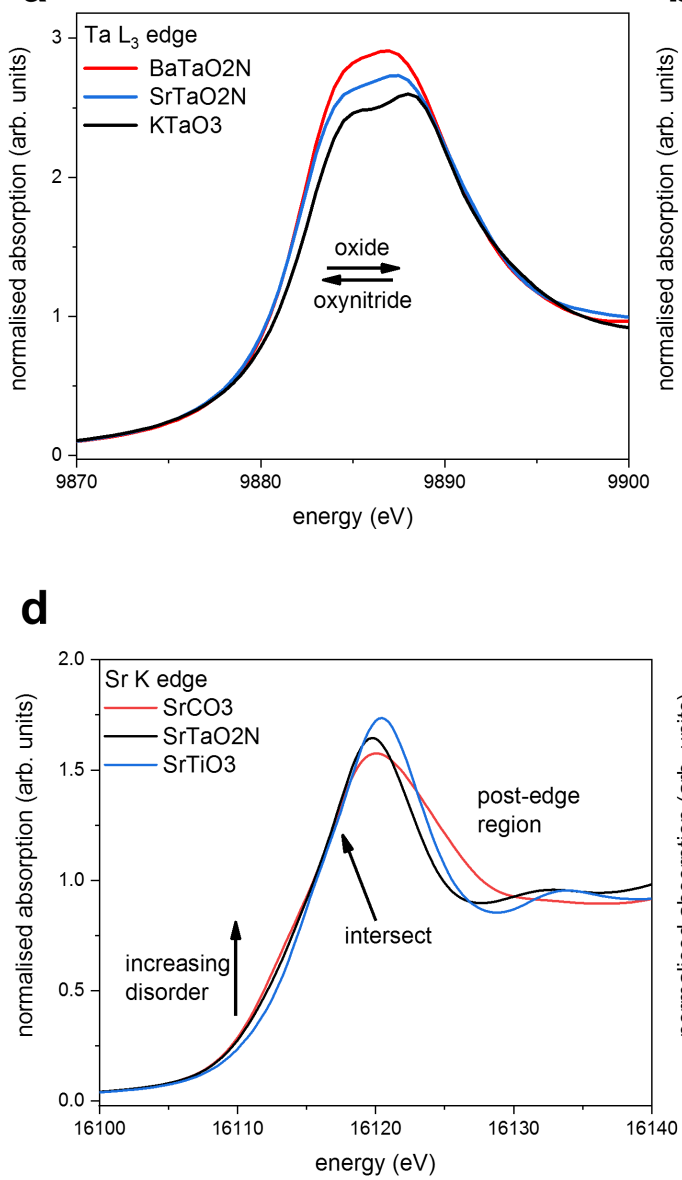

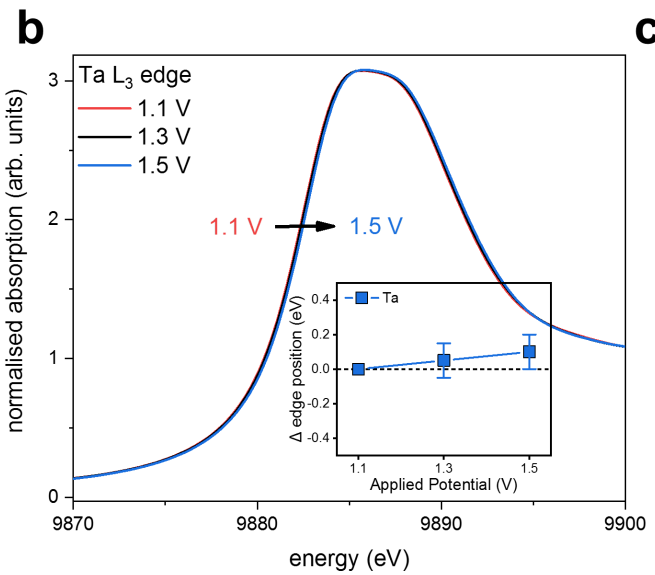

e

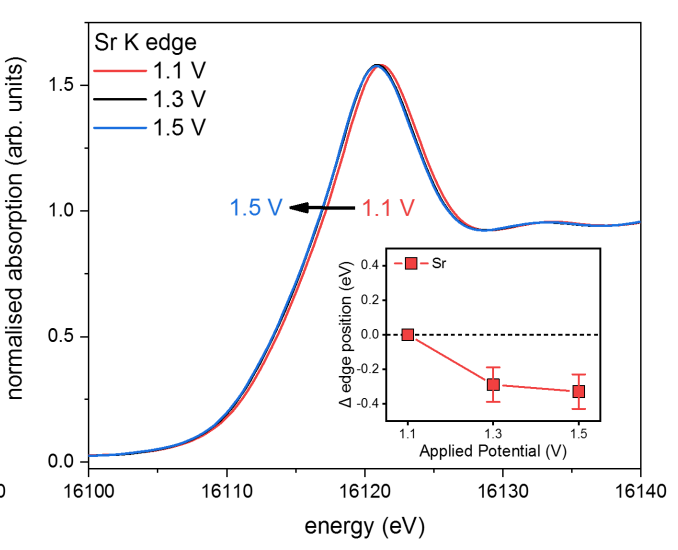

C

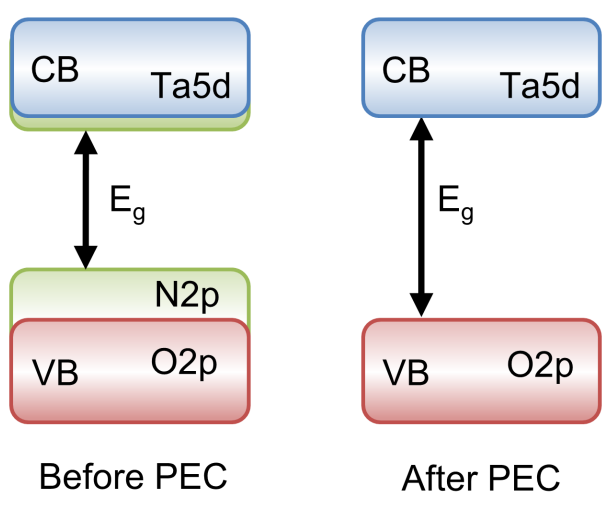

f

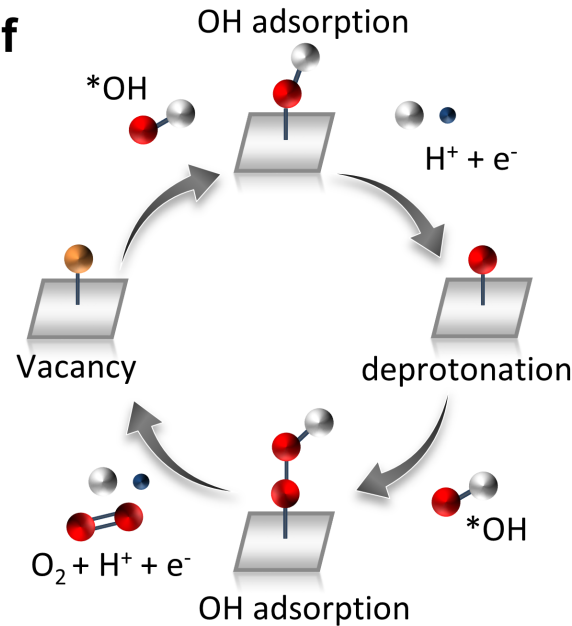

Adsorbed $\mathrm{OOH}$ species

\section{Figure 5}

Operando GIXAS at the STON-liquid interface. (a) Reference spectra for Ta5+ oxide and oxynitride powders, (b) operando measurements for STON thin film during the chronoamperometry (1 hour) measurements, with stepped applied potential. The error bars included in the magnified insert correspond to $\pm 0.1 \mathrm{eV}$, (c) graphic representation for the suggested changes occurring to the band structure of the surface layers of the STON photocatalyst. VB, CB and Eg denote the valence band, conduction band and band gap, respectively, (d) reference spectra for Sr containing powders, (e) operando measurements for STON thin film during the chronoamperometry (1 hour) measurements, with stepped applied potential. The error bars included in the magnified insert correspond to $\pm 0.1 \mathrm{eV}$, (f) suggested changes with respect to Sr after PEC. 
a

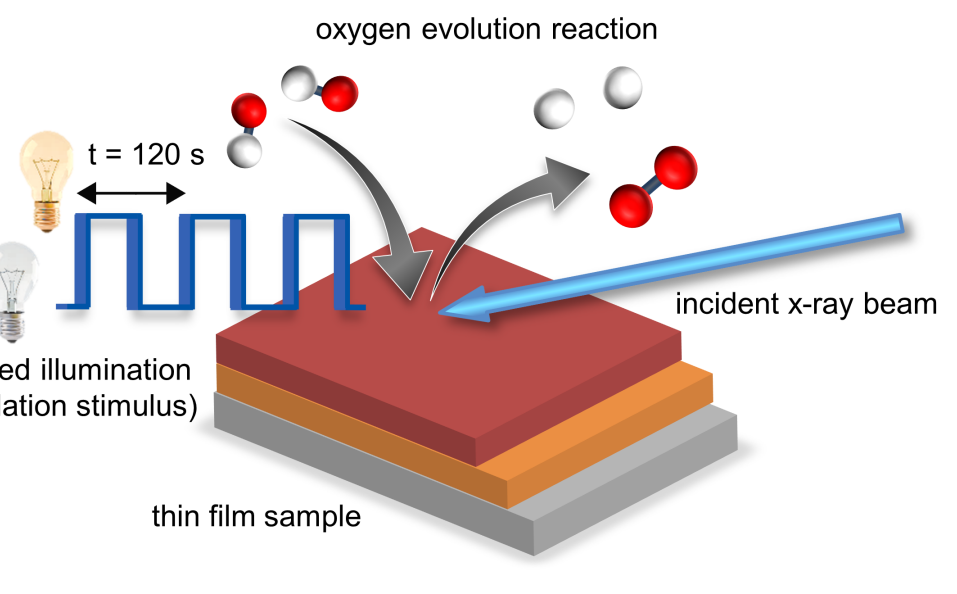

c

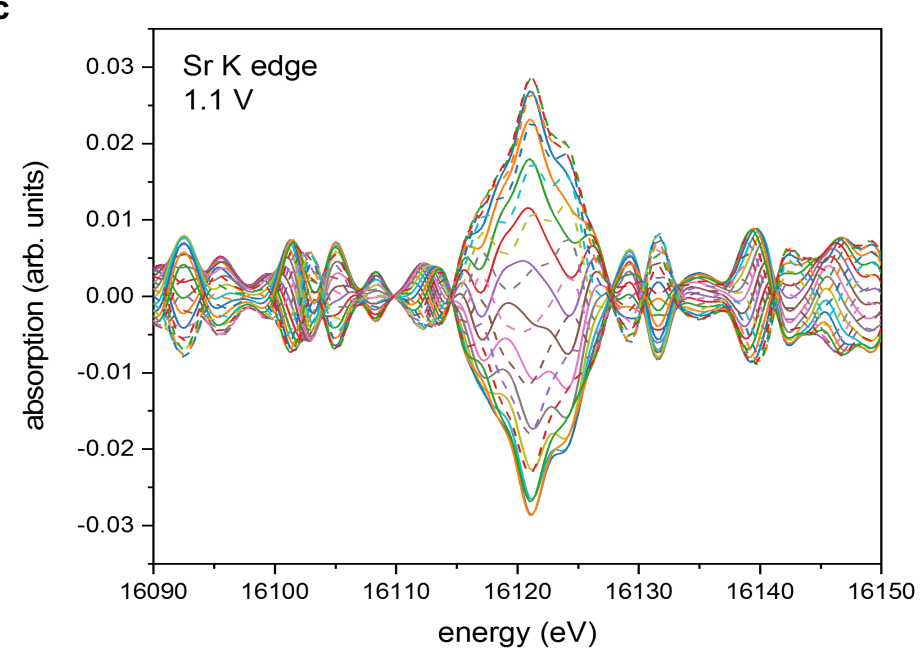

b

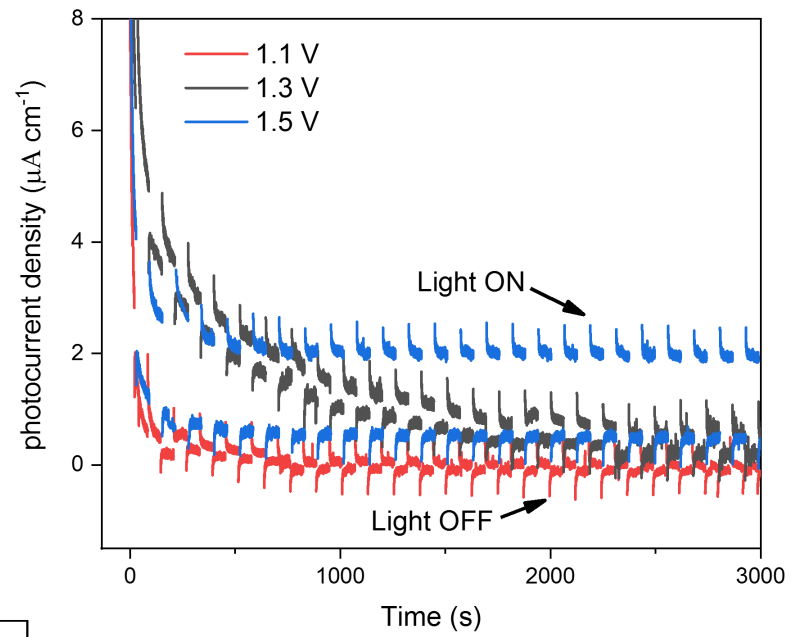

$-\varphi=0^{\circ}-\varphi=180^{\circ}$

$-\varphi=15^{\circ}--\varphi=195^{\circ}$

$-\varphi=30^{\circ}-\cdots=210^{\circ}$

$-\varphi=45^{\circ}--\varphi=225^{\circ}$

$-\varphi=60^{\circ}-\varphi \varphi=240^{\circ}$

$-\varphi=75^{\circ}--\varphi=255^{\circ}$

$-\varphi=90^{\circ}--\varphi=270^{\circ}$

$\varphi=105^{\circ}--\varphi=285^{\circ}$

$\varphi=120^{\circ}--\varphi=300^{\circ}$

$-\varphi=135^{\circ}-\varphi=315^{\circ}$

$-\varphi=150^{\circ}--\varphi=330$

$-\varphi=165^{\circ}--\varphi=345^{\circ}$

d

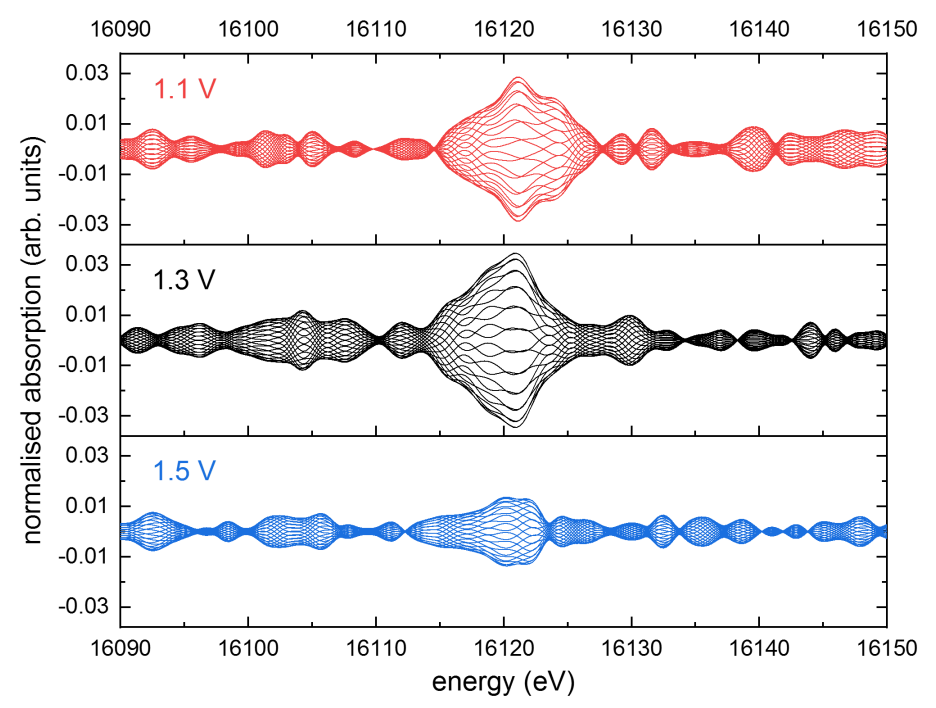

e

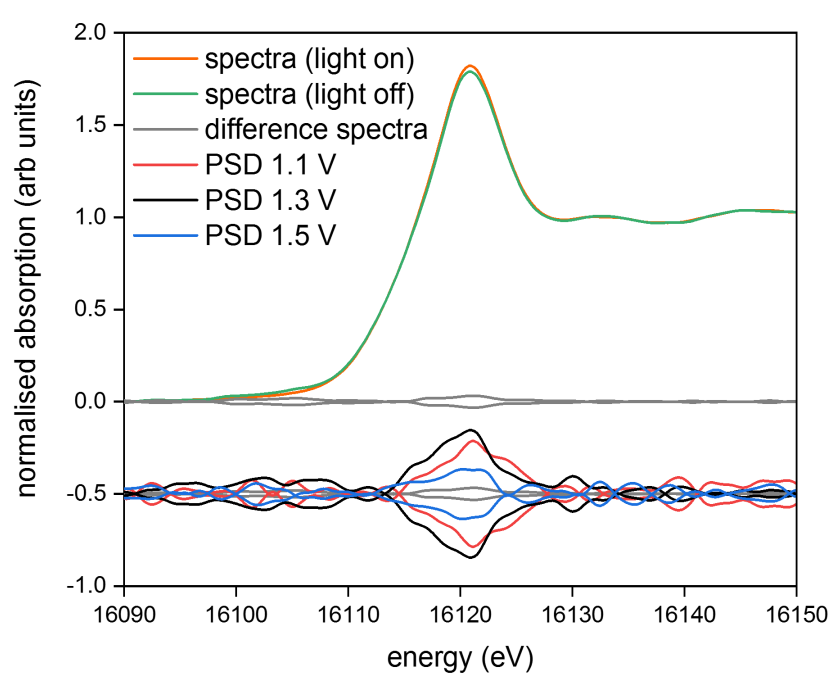

\section{Figure 6}

Operando ME-XAS at the STON-liquid interface. (a) schematic of the experiment, (b) chronoamperometry measurements performed at $1.1 \mathrm{~V}, 1.3 \mathrm{~V}$ and $1.5 \mathrm{~V}$ vs. RHE for 60 minutes under chopped illumination with 60 second periods, (c) phase resolved spectra ( $\triangle \mathrm{PSD}=0^{\circ}-345^{\circ}$ ) obtained from the modulation experiment measured at $1.1 \mathrm{~V}$ at the $\mathrm{Sr} \mathrm{K}$ edge, (d) phase resolved spectra for $\mathrm{Sr} \mathrm{K}$ edge measured at 1.1 $\mathrm{V}, 1.3 \mathrm{~V}$ and $1.5 \mathrm{~V}$ vs. RHE shown in dark grey, light grey and black respectively, (e) average of spectra 
recorded under illumination and under dark conditions, with the difference and PSD spectra at each potential.

\section{Supplementary Files}

This is a list of supplementary files associated with this preprint. Click to download.

- NCoperandoXASsupplementary.pdf 